\title{
Quantum and private capacities of low-noise channels
}

\author{
Felix Leditzky*a,b ${ }^{*}$ Debbie Leung ${ }^{\dagger c}$, and Graeme Smith ${ }^{\ddagger a, b, d}$ \\ a JILA, University of Colorado/NIST, 440 UCB, Boulder, CO 80309, USA \\ ${ }^{\mathrm{b}}$ Center for Theory of Quantum Matter, University of Colorado, Boulder, Colorado 80309, USA \\ 'Institute for Quantum Computing, University of Waterloo, Waterloo, Ontario, Canada, N2L 3G1 \\ ${ }^{\mathrm{d} D e p a r t m e n t}$ of Physics, University of Colorado, 390 UCB, Boulder, CO 80309, USA
}

September 25, 2017

\begin{abstract}
We determine both the quantum and the private capacities of low-noise quantum channels to leading orders in the channel's distance to the perfect channel. It has been an open problem for more than 20 years to determine the capacities of some of these low-noise channels such as the depolarizing channel.

We also show that both capacities are equal to the single-letter coherent information of the channel, again to leading orders. We thus find that, in the low noise regime, super-additivity and degenerate codes have negligible benefit for the quantum capacity, and shielding does not improve the private capacity beyond the quantum capacity, in stark contrast to the situation when noisier channels are considered.
\end{abstract}

\section{Introduction}

Any point-to-point communication link can be modeled as a quantum channel $\mathcal{N}$ from a sender to a receiver. Of fundamental interest are the capacities of $\mathcal{N}$ to transmit data of various types such as quantum, private, or classical data. Informally, the capacity of $\mathcal{N}$ to transmit a certain type of data is the optimal rate at which that data can be transmitted with high fidelity given an asymptotically large number of uses of $\mathcal{N}$. Capacities of a channel quantify its value as a communication resource.

In the classical setting, the capacity of a classical channel $\mathcal{N}$ to transmit classical data is given by Shannon's noisy coding theorem [20]. While operationally, the capacity-achieving error correcting codes may have increasingly large block lengths, the capacity can be expressed as a single letter formula: it is the maximum correlation between input and output that can be generated with a single channel use, where correlation is measured by the mutual information.

In the quantum setting, the capacity of a quantum channel $\mathcal{N}$ to transmit quantum data, denoted $Q(\mathcal{N})$, is given by the LSD theorem [17, 21,4]. A capacity expression is found, but it involves a quantity optimized over an unbounded number of uses of the channel. This quantity, when optimized over $n$ channel uses, is called the $n$-shot coherent information. Dividing the $n$-shot coherent information by $n$ and taking the limit $n \rightarrow \infty$ gives the capacity. For special channels called

\footnotetext{
*Email: felix.leditzky@jila.colorado.edu

$\dagger$ Email: wcleung@uwaterloo.ca

${ }^{\ddagger}$ Email: gsbsmith@gmai l . com
} 
degradable channels, the coherent information is weakly additive, meaning that the $n$-shot coherent information is $n$ times the 1-shot coherent information [5], hence the capacity is the 1-shot coherent information and can be evaluated in principle. In general, the coherent information can be superadditive, meaning that the $n$-shot coherent information can be more than $n$ times the 1 -shot coherent information, thus the optimization over $n$ is necessary [6]. Consequently, there is no general algorithm to compute the capacity of a given channel. Furthermore, the $n$-shot coherent information can be positive for some small $n$ while the 1-shot coherent information is zero [6]. Moreover, given any $n$, there is a channel whose $n$-shot coherent information is zero but whose quantum capacity is positive [3]. Thus we do not have a general method to determine if a given channel has positive quantum capacity.

Even for the qubit depolarizing channel, which acts as $\mathcal{D}_{p}(\rho)=\left(1-\frac{4 p}{3}\right) \rho+\frac{4 p}{3} \frac{I}{2}$, our understanding of the quantum capacity is limited. For $p=0$ the channel is perfect, so we have $Q\left(\mathcal{D}_{0}\right)=1$, while for $p \geq 1 / 4$, we know that $Q\left(\mathcal{D}_{p}\right)=0$ [1]. However, for $0<p<1 / 4$ the quantum capacity of $\mathcal{D}_{p}$ is unknown despite substantial effort (see e.g. [24, 8, 13]). For $p \approx 0.2$, communication rates higher than the 1-shot coherent information are achievable $[6,24,8]$, but even the threshold value of $p$ where the capacity goes to zero is unknown. For $p$ close to zero, the best lower bound for $Q\left(\mathcal{D}_{p}\right)$ is the one-shot coherent information. In this regime, the continuity bound developed in [14] is insufficient to constrain the quantum capacity of $\mathcal{D}_{p}$ to the leading order in $p$, and while various other upper bounds exist, they all differ from the one-shot coherent information by $O(p)$. Recently, a numerical upper bound on the capacity of the low-noise depolarizing channel [29] was found to be very close to the 1-shot coherent information. Meanwhile, the complementary channel for the depolarizing channel for any $p>0$ is found to always have positive capacity [13], which renders several techniques inapplicable, including those described in [30] or a generalization of degradability to "information degradability" [2].

In this paper, we consider the quantum capacity of "low-noise quantum channels" that are close to the identity channel, and investigate how close the capacity is to the 1-shot coherent information. It has been unclear whether we should expect substantial nonadditivity of coherent information for such channels. On the one hand, all known degenerate codes that provide a boost to quantum capacity first encode one logical qubit into a small number of physical qubits, which incurs a significant penalty in rate. This would seem to preclude any benefit in the regime where the 1shot coherent information is already quite high. On the other hand, we have no effective methods for evaluating the $n$-letter coherent information for large $n$, and there may well exist new types of coding strategies that incur no such penalty in the large $n$ regime.

We prove in this paper that to linear order in the noise parameter, the quantum capacity of any low-noise channel is its 1-shot coherent information (see Theorem 12). Consequently, degenerate codes cannot improve the rates of these channels up to the same order. For the special cases of the qubit depolarizing channel, the mixed Pauli channel and their qudit generalizations, we show that the quantum capacity and the 1-shot coherent information agree to even higher order (see Theorem 14).

Our findings extend to the private capacity $P(\mathcal{N})$ of a quantum channel $\mathcal{N}$, which displays similar complexities to the quantum capacity. The private capacity is equal to the regularized private information [4], but the private information is not additive $([23,16,25])$. In [10], the private capacity, which is never smaller than the quantum capacity, is found to be positive for some channels that have no quantum capacity. The authors also characterize the type of noise that hurts quantum transmission and that can be "shielded" from corrupting private data. In [15], channels are found with almost no quantum capacity but maximum private capacity. Meanwhile, for degrad- 
able channels, the private capacity is again equal to the 1-shot coherent information [22]. This coincidence of $P$ and $Q$ for degradable channels means that our findings for the quantum capacity can be carried over to private capacity fairly easily. In the low-noise regime the private capacity is also equal to the 1-shot coherent information to linear order in the noise parameter, and is equal to the quantum capacity in the same order (see Theorems 12 and 14). Consequently, shielding provides little benefit.

Our results follow closely the approach in [29]. Consider a channel $\mathcal{N}$ and its complementary channel $\mathcal{N}^{c}$. The channel $\mathcal{N}$ is degradable if there is another channel $\mathcal{M}$ (called a degrading map) such that $\mathcal{M} \circ \mathcal{N}=\mathcal{N}^{c}$. Instead of measuring how close $\mathcal{N}$ is to some degradable channel, [29] considers how close $\mathcal{M} \circ \mathcal{N}$ can be to $\mathcal{N}^{c}$ when optimizing over all channels $\mathcal{M}$, a measure we call the degradability parameter of $\mathcal{N}$. Furthermore, this distance between $\mathcal{N}^{c}$ and $\mathcal{M} \circ \mathcal{N}$ as well as the best approximate degrading map $\mathcal{M}$ can be obtained via semidefinite programming. Continuity results, relative to the case as if $\mathcal{N}$ is degradable, can then be obtained similarly to [14]. This new bound in [29] limits the difference between the 1-shot coherent information and the quantum capacity to $O(\eta \log \eta)$ where $\eta$ is the degradability parameter. Note that $\eta \log \eta$ does not have a finite slope at $\eta=0$ but it goes to zero faster than $\eta^{b}$ for any $b<1$. While this method does not yield explicit upper bounds, once a channel of interest is fixed, it is fairly easy to evaluate the degradability parameter (via semidefinite programming) and the resulting capacity bounds numerically.

The primary contribution in this paper is an analytic proof of a surprising fact that, for low-noise channels whose diamond-norm distance to being noiseless is $\varepsilon$, the degradability parameter $\eta$ grows at most as fast as $O\left(\varepsilon^{1.5}\right)$, rendering the gap $O(\eta \log \eta)$ between the 1-shot coherent information and the quantum or private capacity only sublinear in $\varepsilon$ (see Theorem 11). For the qubit depolarizing channel and its various generalizations, we improve the analytic bound of $\eta$ to $O\left(\varepsilon^{2}\right)$ (see Theorem 13). Furthermore, for both results, we provide constructive approximate degrading maps and explain why they work well.

The rest of the paper is structured as follows. In Section 2, we explain both our notations and prior results relevant to our discussion. We present our results for a general low-noise channel in Section 3 and for the depolarizing channel in Section 4.1 (with the various generalizations in Section 4.2). We conclude with some extensions and implications of our results in Section 5.

\section{Background}

In this paper we only consider finite-dimensional Hilbert spaces. For a Hilbert space $\mathcal{H}$, we denote by $\mathcal{B}(\mathcal{H})$ the set of linear operators on $\mathcal{H}$. We write $M_{A}$ for operators defined on a Hilbert space $\mathcal{H}_{A}$ associated with a quantum system $A$. We denote by $I_{A}$ the identity operator on $\mathcal{H}_{A}$, and by $\operatorname{id}_{A}$ the identity map on $\mathcal{B}\left(\mathcal{H}_{A}\right)$. We denote the dimension of $A$ by $|A|=\operatorname{dim} \mathcal{H}_{A}$. A (quantum) state $\rho_{A}$ on a quantum system $A$ is a positive semidefinite operator with unit trace, that is, $\rho_{A} \geq 0$ and $\operatorname{tr} \rho_{A}=1$.

\subsection{Quantum channels}

Any point-to-point communication link that transmits an input to an output state can be modeled as a quantum channel. Mathematically, a quantum channel is a linear, completely positive, and trace-preserving map $\mathcal{N}$ from $\mathcal{B}\left(\mathcal{H}_{A}\right)$ to $\mathcal{B}\left(\mathcal{H}_{B}\right)$. We often use the shorthand $\mathcal{N}: A \rightarrow B$. If a 
channel $\mathcal{N}^{\prime}: A \rightarrow B^{\prime}$ acts as $\mathcal{N}$ followed by an isometry from $B$ to $B^{\prime}$, then we call $\mathcal{N}^{\prime}$ equivalent to $\mathcal{N}$. This is an equivalence relation on the set of quantum channels, and for a given channel $\mathcal{N}$ all analysis of interest in this paper applies to any channel in the equivalence class of $\mathcal{N}$. We summarize two useful representations for quantum channels in the following [18, 33].

For every quantum channel $\mathcal{N}: A \rightarrow B$ there is an isometric extension $V: \mathcal{H}_{A} \rightarrow \mathcal{H}_{B} \otimes \mathcal{H}_{E}$ for some auxiliary Hilbert space $\mathcal{H}_{E}$ associated with some environment system $E$, such that $\mathcal{N}\left(\rho_{A}\right)=$ $\operatorname{tr}_{E}\left(V \rho_{A} V^{\dagger}\right)$ [27]. The isometric extension is also called the Stinespring dilation, and this representation is sometimes called the unitary representation. Note that the isometric extension is unique up to left multiplication by some unitary on $E$, and this degree of freedom does not affect most analysis of interest. Physically, the isometric extension distributes the input to $B$ and $E$ jointly, and the channel $\mathcal{N}$ is noisy because the information in $E$ is no longer accessible. The complementary channel $\mathcal{N}^{c}: A \rightarrow E$ of $\mathcal{N}$ with respect to a specific isometric extension $V$ is defined by $\mathcal{N}^{c}\left(\rho_{A}\right):=\operatorname{tr}_{B}\left(V \rho_{A} V^{\dagger}\right)$. Note that all channels complementary to $\mathcal{N}$ are equivalent.

The second representation we use is the Choi-Jamiolkowski isomorphism. It is a bijection $\mathcal{J}$ from the set of quantum channels $\mathcal{N}: A \rightarrow B$ to the set of positive operators $\tau_{A^{\prime} B} \in \mathcal{B}\left(\mathcal{H}_{A^{\prime}} \otimes \mathcal{H}_{B}\right)$ satisfying $\operatorname{tr}_{B} \tau_{A^{\prime} B}=I_{A^{\prime}}$, given by:

$$
\mathcal{J}(\mathcal{N}):=\left(\operatorname{id}_{A^{\prime}} \otimes \mathcal{N}\right)\left(\gamma_{A^{\prime} A}\right)
$$

where $|\gamma\rangle_{A^{\prime} A}:=\sum_{i}|i\rangle_{A^{\prime}} \otimes|i\rangle_{A}$ is proportional to the maximally entangled state on $A^{\prime} A$, the system $A^{\prime}$ is isomorphic to $A$, and $\gamma_{A^{\prime} A}=|\gamma\rangle\left\langle\left.\gamma\right|_{A^{\prime} A}\right.$. The inverse of $\mathcal{J}$ applied to $\tau_{A^{\prime} B}$ yields a channel whose action on an operator $\rho_{A}$ defined on a system $A$ is given by

$$
\left(\mathcal{J}^{-1}\left(\tau_{A^{\prime} B}\right)\right)\left(\rho_{A}\right)=\operatorname{tr}_{A^{\prime}}\left(\tau_{A^{\prime} B}\left(\left(\rho^{T}\right)_{A^{\prime}} \otimes \mathbb{1}_{B}\right)\right)
$$

where $T$ denotes transposition with respect to the basis $\{|i\rangle\}$ that defines $|\gamma\rangle$. The operator $\mathcal{J}(\mathcal{N})$ is called the Choi matrix of $\mathcal{N}$. It is uniquely determined by $\mathcal{N}$ and it is basis-dependent. The rank of $\mathcal{J}(\mathcal{N})$ is called the Choi rank of $\mathcal{N}$. It is the minimum dimension of the environment $E$ for an isometric extension of $\mathcal{N}$. It is basis-independent, and independent of the choice of the isometric extension.

\subsection{The diamond norm and the continuity of the isometric extension}

For an operator $M \in \mathcal{B}(\mathcal{H})$ we define the trace norm $\|M\|_{1}$, the operator norm $\|M\|_{\infty}$, and the max norm $\|M\|_{\max }$ as follows:

$$
\begin{aligned}
\|M\|_{1} & :=\operatorname{tr} \sqrt{M^{+} M} \\
\|M\|_{\infty} & :=\max \left\{\sqrt{\left\langle\psi\left|M^{\dagger} M\right| \psi\right\rangle}:|\psi\rangle \in \mathcal{H},\langle\psi \mid \psi\rangle=1\right\}, \\
\|M\|_{\max } & :=\max _{i, j}\left|M_{i, j}\right| .
\end{aligned}
$$

Note that the max norm is basis-dependent, unlike the trace and the operator norms.

We now discuss the distance measure we use for channels. For a linear map $\Phi: \mathcal{B}(\mathcal{H}) \rightarrow \mathcal{B}(\mathcal{K})$ between Hilbert spaces $\mathcal{H}$ and $\mathcal{K}$, we define its diamond norm by

$$
\|\Phi\|_{\diamond}:=\max \left\{\left\|\left(\operatorname{id}_{\mathbb{C}^{n}} \otimes \Phi\right)(M)\right\|_{1}: M \in \mathcal{B}\left(\mathbb{C}^{n} \otimes \mathcal{H}\right),\|M\|_{1}=1, n \in \mathbb{N}\right\},
$$


where $\operatorname{id}_{\mathbb{C}^{n}}$ denotes the identity map on $\mathbb{C}^{n}$. It suffices to take $n$ as large as $\operatorname{dim}(\mathcal{H})$ in the above optimization so that the maximum can be attained. When applied to the difference of two channels $\mathcal{N}_{1}-\mathcal{N}_{2}$, the diamond norm has a simple operational meaning: it is twice the maximum of the trace distance of the two output states $\left(\operatorname{id}_{\mathbb{C}^{n}} \otimes \mathcal{N}_{i}\right)(M)$ (for $\left.i=1,2\right)$ created by the two channels on the best common input $M$, in the presence of an ancillary space $\mathbb{C}^{n}$. The trace distance of two states in turn signifies their distinguishability [9]. We summarize two characterizations of the diamond norm, a method to upper bound it, and a continuity result for the isometric extension in the rest of this subsection.

First, the diamond norm $\left\|\mathcal{N}_{1}-\mathcal{N}_{2}\right\|_{\diamond}$ of the difference of two quantum channels $\mathcal{N}_{1}: A \rightarrow B$ and $\mathcal{N}_{2}: A \rightarrow B$ can be computed by solving the following semidefinite program (SDP) [31]:

$$
\begin{aligned}
\text { minimize: } & 2 \mu \\
\text { subject to: } & \operatorname{tr}_{B} Z_{A B} \leq \mu I_{A} \\
& Z_{A B} \geq \mathcal{J}\left(\mathcal{N}_{1}\right)-\mathcal{J}\left(\mathcal{N}_{2}\right) \\
& Z_{A B} \geq 0
\end{aligned}
$$

Second, the diamond norm of a linear CP map $\Theta$ (which is not necessarily trace-preserving) can be rewritten as ([32] Thm. 6):

$$
\|\Theta\|_{\diamond}=\max _{\rho_{1}, \rho_{2}}\left\{\left\|\left(\sqrt{\rho_{1}} \otimes \mathbb{1}_{B}\right) \mathcal{J}(\Theta)\left(\sqrt{\rho_{2}} \otimes \mathbb{1}_{B}\right)\right\|_{1}\right\},
$$

where the maximum is over density matrices $\rho_{1}$ and $\rho_{2}$ on the input system $A$ of the map $\Theta$.

We prove the following technical lemma that upper bounds the diamond norm of an arbitrary linear CP map (which need not be trace preserving) using the max norm of its Choi matrix:

Lemma 1. For a linear $C P$ map $\Theta: A \rightarrow B$, we have $\|\Theta\|_{\diamond} \leq|A||B|^{2}\|\mathcal{J}(\Theta)\|_{\max }$.

Proof. We start with the second characterization of the diamond norm in (3) above, and let $\sigma_{1}$ and $\sigma_{2}$ be states on $A$ achieving the maximum in (3), such that

$$
\|\Theta\|_{\diamond}=\left\|\left(\sqrt{\sigma_{1}} \otimes \mathbb{1}_{B}\right) \mathcal{J}(\Theta)\left(\sqrt{\sigma_{2}} \otimes \mathbb{1}_{B}\right)\right\|_{1} .
$$

Furthermore, we employ the fact that the trace distance can be expressed as [18]

$$
\|M\|_{1}=\max _{U}|\operatorname{tr}(U M)|
$$

where the maximum is over unitary operators $U$. Let $V$ be a unitary operator achieving the above maximum in (4). We then have

$$
\begin{aligned}
&\|\Theta\|_{\diamond}=\left|\operatorname{tr}\left(V\left(\sqrt{\sigma_{1}} \otimes \mathbb{1}_{B}\right) \mathcal{J}(\Theta)\left(\sqrt{\sigma_{2}} \otimes \mathbb{1}_{B}\right)\right)\right| \\
&=\left|\operatorname{tr}\left(\sqrt{V}\left(\sqrt{\sigma_{1}} \otimes \mathbb{1}_{B}\right) \sqrt{\mathcal{J}(\Theta)} \sqrt{\mathcal{J}(\Theta)}\left(\sqrt{\sigma_{2}} \otimes \mathbb{1}_{B}\right) \sqrt{V}\right)\right| \\
& \leq \sqrt{\operatorname{tr}\left(V^{1 / 2}\left(\sqrt{\sigma_{1}} \otimes \mathbb{1}_{B}\right) \mathcal{J}(\Theta)\left(\sqrt{\sigma_{1}} \otimes \mathbb{1}_{B}\right) V^{-1 / 2}\right)} \\
& \quad \times \sqrt{\operatorname{tr}\left(V^{-1 / 2}\left(\sqrt{\sigma_{2}} \otimes \mathbb{1}_{B}\right) \mathcal{J}(\Theta)\left(\sqrt{\sigma_{2}} \otimes \mathbb{1}_{B}\right) V^{1 / 2}\right)} \\
&=\sqrt{\operatorname{tr}\left(\left(\sigma_{1} \otimes \mathbb{1}_{B}\right) \mathcal{J}(\Theta)\right)} \sqrt{\operatorname{tr}\left(\left(\sigma_{2} \otimes \mathbb{1}_{B}\right) \mathcal{J}(\Theta)\right)}
\end{aligned}
$$




$$
\begin{aligned}
& =|B| \sqrt{\operatorname{tr}\left(\left(\sigma_{1} \otimes \pi_{B}\right) \mathcal{J}(\Theta)\right)} \sqrt{\operatorname{tr}\left(\left(\sigma_{2} \otimes \pi_{B}\right) \mathcal{J}(\Theta)\right)} \\
& \leq|B|\|\mathcal{J}(\Theta)\|_{\infty},
\end{aligned}
$$

where $\pi_{B}=I_{B} /|B|$, and we have used the Cauchy-Schwarz inequality for the Hilbert-Schmidt inner product $\langle X, Y\rangle:=\operatorname{tr}\left(X^{\dagger} Y\right)$ in the first inequality, and the identity

$$
\|Z\|_{\infty}=\max \{|\operatorname{tr}(\rho Z)|: \rho \geq 0, \operatorname{tr} \rho=1\}
$$

in the second inequality. To bound the operator norm of $J \equiv \mathcal{J}(\Theta)$ in (5), let $n=|A||B|$ and $\lambda_{1} \geq \cdots \geq \lambda_{n}$ be the eigenvalues of $J$ in descending order, and observe that we have

$$
\|J\|_{\infty}^{2}=\lambda_{1}^{2} \leq \sum_{k=1}^{n} \lambda_{k}^{2}=\operatorname{tr}\left(J^{2}\right)=\sum_{i, j=1}^{n} J_{i j} J_{j i}=\sum_{i, j=1}^{n}\left|J_{i j}\right|^{2} \leq n^{2}\|J\|_{\max }^{2} .
$$

Hence, $\|\mathcal{J}(\Theta)\|_{\infty} \leq|A||B|\|\mathcal{J}(\Theta)\|_{\max }$, which together with (5) proves the claim.

Corollary 2. If $\Theta$ is a CP map whose Choi matrix has coefficients $O\left(p^{2}\right)$ for $p$ in a neighborhood of 0 , then also $\|\Theta\|_{\diamond}=O\left(p^{2}\right)$.

We also use the following continuity result for the isometric extensions for channels [11] :

Theorem 3 ([11]). For quantum channels $\mathcal{N}_{1}$ and $\mathcal{N}_{2}$,

$$
\inf _{V_{1}, V_{2}}\left\|V_{1}-V_{2}\right\|_{\infty}^{2} \leq\left\|\mathcal{N}_{1}-\mathcal{N}_{2}\right\|_{\diamond} \leq 2 \inf _{V_{1}, V_{2}}\left\|V_{1}-V_{2}\right\|_{\infty}
$$

where the infimum is over isometric extensions $V_{i}$ of $\mathcal{N}_{i}$ for $i=1,2$, respectively.

A simple consequence of Theorem 3 is that for two quantum channels that are close in the diamond norm, their complementary channels can be made similarly close to one another:

Corollary 4. Let $\mathcal{N}_{1}, \mathcal{N}_{2}$ be quantum channels with $\left\|\mathcal{N}_{1}-\mathcal{N}_{2}\right\|_{\diamond} \leq \varepsilon$ for some $\varepsilon \in[0,2]$. Then for any complementary channel $\mathcal{N}_{1}^{c}$ of $\mathcal{N}_{1}$, there exists a complementary channel $\mathcal{N}_{2}^{c}$ of $\mathcal{N}_{2}$ such that

$$
\left\|\mathcal{N}_{1}^{c}-\mathcal{N}_{2}^{c}\right\|_{\diamond} \leq 2 \sqrt{\varepsilon}
$$

\subsection{Quantum and private capacities and approximate degradability}

The coherent information of a state $\rho_{A}$ through a channel $\mathcal{N}: A \rightarrow B$ is defined as

$$
I_{c}\left(\rho_{A} ; \mathcal{N}\right):=S\left(\mathcal{N}\left(\rho_{A}\right)\right)-S\left(\mathcal{N}^{c}\left(\rho_{A}\right)\right),
$$

where $S(\sigma):=-\operatorname{tr} \sigma \log \sigma$ denotes the von Neumann entropy of $\sigma$, and log is base 2 throughout this paper. Note that the coherent information is independent of the choice of the complementary channel. The coherent information can be interpreted as follows. Let $|\psi\rangle_{A^{\prime} A}$ be a purification of $\rho_{A}$ (that is, $\operatorname{tr}_{A^{\prime}}|\psi\rangle\left\langle\left.\psi\right|_{A^{\prime} A}=\rho_{A}\right)$. Then, $I_{c}\left(\rho_{A} ; \mathcal{N}\right)=\frac{1}{2}\left(I\left(A^{\prime}: B\right)-I\left(A^{\prime}: E\right)\right)$ where $I\left(A^{\prime}: B\right)=$ $S\left(A^{\prime}\right)+S(B)-S\left(A^{\prime} B\right)$ is the quantum mutual information between $A^{\prime}$ and $B$, and similarly for $I\left(A^{\prime}: E\right)$, and the mutual information is evaluated on the state $\operatorname{id}_{A^{\prime}} \otimes \mathcal{N}\left(|\psi\rangle\left\langle\left.\psi\right|_{A^{\prime} A}\right)\right.$. The coherent information of $\mathcal{N}$, also called the 1-shot coherent information, is the maximum over all input states,

$$
I_{\mathcal{C}}(\mathcal{N}):=\max _{\rho_{A}} I_{\mathcal{C}}\left(\rho_{A} ; \mathcal{N}\right)
$$


The $n$-shot coherent information of $\mathcal{N}$ is defined as $I_{c}^{(n)}(\mathcal{N}):=I_{\mathcal{c}}\left(\mathcal{N}^{\otimes n}\right)$, and satisfies $I_{c}^{(n+m)}(\mathcal{N}) \geq$ $I_{c}^{(n)}(\mathcal{N})+I_{c}^{(m)}(\mathcal{N})$ for $n, m \in \mathbb{N}$. The quantum capacity theorem $[17,21,4]$ establishes that the quantum capacity of $\mathcal{N}$ is given by the following regularized formula:

$$
Q(\mathcal{N})=\lim _{n \rightarrow \infty} \frac{1}{n} I_{c}^{(n)}(\mathcal{N})=\sup _{n \in \mathbb{N}} \frac{1}{n} I_{c}^{(n)}(\mathcal{N}),
$$

where the second equality follows from Fekete's lemma [7]. In general, the regularization in (6) is necessary, and renders the quantum capacity intractable to compute for most channels [6,3]. However, for the class of degradable channels [5], the formula (6) reduces to a single-letter formula. A channel $\mathcal{N}$ with complementary channel $\mathcal{N}^{c}$ is degradable if there is another channel $\mathcal{M}$ (called a degrading map) such that $\mathcal{M} \circ \mathcal{N}=\mathcal{N}^{c}$. For degradable channels, the coherent information is weakly additive, $I_{c}^{(n)}(\mathcal{N})=n I_{\mathcal{c}}(\mathcal{N})$ [5]. As a result, the limit in (6) is unnecessary, and we have

$$
Q(\mathcal{N})=I_{c}(\mathcal{N})
$$

Moreover, for a degradable channel $\mathcal{N}$ the coherent information $I_{\mathcal{c}}\left(\rho_{A} ; \mathcal{N}\right)$ is concave in the input state $\rho_{A}$ [34], and thus $I_{\mathcal{C}}(\mathcal{N})$ can be efficiently computed using standard optimization techniques.

Since degradable channels have nice properties that allow us to determine their quantum capacity, we might ask if some of these properties are approximately satisfied by channels that are "almost" degradable. Reference [29] formalized this idea by considering how close $\mathcal{M} \circ \mathcal{N}$ can be made to $\mathcal{N}^{c}$ when optimizing over the channel $\mathcal{M}$.

Definition 5 ([29]; Approximate degradability). A quantum channel $\mathcal{N}: A \rightarrow B$ with environment $E$ is called $\eta$-degradable for an $\eta \in[0,2]$ if there exists a quantum channel $\mathcal{M}: B \rightarrow E$ such that

$$
\left\|\mathcal{N}^{c}-\mathcal{M} \circ \mathcal{N}\right\|_{\diamond} \leq \eta
$$

The degradability parameter $\operatorname{dg}(\mathcal{N})$ of $\mathcal{N}$ is defined as the minimal $\eta$ such that (7) holds for some quantum channel $\mathcal{M}: B \rightarrow E$, and $\mathcal{N}$ is degradable if $\operatorname{dg}(\mathcal{N})=0$.

Note that every quantum channel is 2-degradable, since $\left\|\mathcal{N}_{1}-\mathcal{N}_{2}\right\|_{\diamond} \leq 2$ for any two quantum channels $\mathcal{N}_{1}$ and $\mathcal{N}_{2}$. The SDP (2) for the diamond norm can be used to express the degradability parameter $\operatorname{dg}(\mathcal{N})$ of Definition 5 as the solution of the following SDP [29]:

$$
\begin{aligned}
\text { minimize: } & 2 \mu \\
\text { subject to: } & \operatorname{tr}_{E} Z_{A E} \leq \mu I_{A} \\
& \operatorname{tr}_{E} Y_{B E}=\mathbb{1}_{B} \\
& Z_{A E} \geq \mathcal{J}\left(\mathcal{N}^{c}\right)-\mathcal{J}\left(\mathcal{J}^{-1}\left(Y_{B E}\right) \circ \mathcal{N}\right) \\
& Z_{A E} \geq 0, Y_{B E} \geq 0 .
\end{aligned}
$$

The bipartite operator $Y_{B E}$ above corresponds to the Choi matrix of the approximate degrading $\operatorname{map} \mathcal{M}: B \rightarrow E$.

While a degradable channel has capacity equal to the 1-shot coherent information, an $\eta$-degradable channel $\mathcal{N}$ has capacity differing from the 1-shot coherent information at most by a vanishing function of $\eta$ : 
Theorem 6 ([29], Thm. $3.3(\mathrm{i})$; Continuity bound). If $\mathcal{N}$ is a channel with degradability parameter $\operatorname{dg}(\mathcal{N})=\eta$, then,

$$
I_{c}(\mathcal{N}) \leq Q(\mathcal{N}) \leq I_{c}(\mathcal{N})+\frac{\eta}{2} \log (|E|-1)+\eta \log |E|+h\left(\frac{\eta}{2}\right)+\left(1+\frac{\eta}{2}\right) h\left(\frac{\eta}{2+\eta}\right),
$$

where $h(x):=-x \log x-(1-x) \log (1-x)$ is the binary entropy function, and $|E|$ is the Choi rank of $\mathcal{N}$.

The private capacity of $\mathcal{N}$, denoted by $P(\mathcal{N})$, is the capacity of $\mathcal{N}$ to transmit classical data with vanishing probability of error such that the state in the joint environment of all channel uses has vanishing dependence on the input. The capacity expression is found to be the regularized private information for $\mathcal{N}$ in [4], but the private information is not additive $([23,16,25])$. For degradable channels, $P(\mathcal{N})=I_{\mathcal{C}}(\mathcal{N})$, and there is a continuity result similar to Theorem 6 :

Theorem 7 ([29], Thm. 3.3 (iii) and (v) combined). If $\mathcal{N}$ is a channel with degradability parameter $\operatorname{dg}(\mathcal{N})=\eta$, then,

$$
I_{\mathcal{C}}(\mathcal{N}) \leq P(\mathcal{N}) \leq I_{\mathcal{c}}(\mathcal{N})+\eta \log (|E|-1)+4 \eta \log |E|+2 h\left(\frac{\eta}{2}\right)+4\left(1+\frac{\eta}{2}\right) h\left(\frac{\eta}{2+\eta}\right)
$$

where $|E|$ and $h$ are as defined in Theorem 6.

\section{General low-noise channel}

Throughout this paper we focus on low-noise channels, by which we mean a channel $\mathcal{N}$ that has isomorphic input and output spaces $A$ and $B$ and approximates the noiseless (or identity) channel in the diamond norm, $\left\|\mathcal{N}-\mathrm{id}_{A}\right\|_{\diamond} \leq \varepsilon$, where $\varepsilon>0$ is a small positive parameter.

\subsection{Deviation of quantum capacity from 1-shot coherent information}

We start from Theorem 6 in Section 2.3, which gives a "continuity bound" on the difference between the quantum capacity and the 1-shot coherent information for an arbitrary channel $\mathcal{N}$ with degradability parameter $\operatorname{dg}(\mathcal{N})=\eta$ :

$$
\left|Q(\mathcal{N})-I_{\mathcal{C}}(\mathcal{N})\right| \leq f_{1}(\eta)
$$

where

$$
f_{1}(\eta):=\frac{\eta}{2} \log (|E|-1)+\eta \log |E|+h\left(\frac{\eta}{2}\right)+\left(1+\frac{\eta}{2}\right) h\left(\frac{\eta}{2+\eta}\right)
$$

satisfying $\lim _{\eta \rightarrow 0} f_{1}(\eta)=0$.

If $\mathcal{N}$ is "almost" degradable, then $\eta \approx 0$. To investigate the behavior of $f_{1}(\eta)$ in this regime, we keep the $\eta \log \eta$ terms in $f_{1}(\eta)$ (knowing that these will dominate for small $\eta$ ), and develop the rest in a Taylor series around 0 . For example, the first binary entropy term is expanded as

$$
h\left(\frac{\eta}{2}\right)=\frac{1}{2}\left(1+(\ln 2)^{-1}-\log \eta\right) \eta-\frac{1}{8}(\ln 2)^{-1} \eta^{2}+O\left(\eta^{3}\right)
$$


The second binary entropy term (including the prefactor) is expanded as

$$
\left(1+\frac{\eta}{2}\right) h\left(\frac{\eta}{2+\eta}\right)=\frac{1}{2}\left(1+(\ln 2)^{-1}-\log \eta\right) \eta+\frac{1}{8}(\ln 2)^{-1} \eta^{2}+O\left(\eta^{3}\right) .
$$

The quadratic contributions cancel out in an expansion of $f_{1}(\eta)$, and including the two linear terms in (9) gives

$$
f_{1}(\eta)=-\eta \log \eta+\left(1+(\ln 2)^{-1}+\frac{1}{2} \log (|E|-1)+\log |E|\right) \eta+O\left(\eta^{3}\right) .
$$

It follows that for small $\eta$ the function $f_{1}(\eta)$ is dominated by $g(\eta):=-\eta \log \eta$ which has infinite slope at $\eta=0$ :

$$
g^{\prime}(\eta)=-\log (\eta)-\frac{1}{\ln 2} \stackrel{\eta \rightarrow 0}{\longrightarrow}+\infty
$$

Hence, without further information on $\eta$, Theorem 6 does not give a tight approximation of the quantum capacity relative to the 1-shot coherent information.

Instead, we consider a scenario in which the channel $\mathcal{N}(p)$ depends on some underlying noise parameter $p \in[0,1]$ and $\eta=\operatorname{dg}(\mathcal{N}(p)) \leq c p^{r}$ where $r>1$ and $c$ is a constant. (Note that $f_{1}(\eta)$ increases with $\eta$ for small $\eta$, so, it suffices to consider $\eta=c p^{r}$.) In this case, the approximation implied by Theorem 6 becomes extremely useful - we will show that the upper bound $I_{c}(\mathcal{N}(p))+$ $f_{1}\left(c p^{r}\right)$ on $Q(\mathcal{N}(p))$ is now tangent to the 1-shot coherent information $I_{\mathcal{c}}(\mathcal{N}(p))$.

Lemma 8. If $r>1$ and $c$ is a constant, then $\left.\frac{d}{d p} f_{1}\left(c p^{r}\right)\right|_{p=0}=0$.

Proof. From (11) and the chain rule, we obtain

$$
\frac{d}{d p} g\left(c p^{r}\right)=\left(-\log \left(c p^{r}\right)-\frac{1}{\ln 2}\right) c r p^{r-1}
$$

Hence, $\lim _{p \rightarrow 0} \frac{d}{d p} g\left(c p^{r}\right)=0$ for any $r>1$. Finally,

$$
\frac{d}{d p} f_{1}\left(c p^{r}\right)=\frac{d}{d p} g\left(c p^{r}\right)+\left(1+(\ln 2)^{-1}+\log |E|+\frac{1}{2} \log (|E|-1)\right) c r p^{r-1}+O\left(p^{3 r-1}\right) .
$$

So, $\left.\frac{d}{d p} f_{1}\left(c p^{r}\right)\right|_{p=0}=0$ as claimed.

Figure 1 and Figure 2 plot $\frac{d}{d p} g\left(c p^{r}\right)$ and $g\left(c p^{r}\right)$, respectively, for the values $c=1$ and $r \in\{1,1.5,2\}$. We also simplify (10) as

Lemma 9. Let $\mathcal{N}=\mathcal{N}(p)$ be a channel defined in terms of a noise parameter $p \in[0,1]$, and $\operatorname{dg}(\mathcal{N}) \leq c p^{r}$ for some $r>1$ and some constant $c$. Then, we have

$$
\begin{aligned}
\left|Q(\mathcal{N})-I_{c}(\mathcal{N})\right| \leq c r p^{r-1}( & -p \log p) \\
& +c p^{r}\left(-\log c+1+(\ln 2)^{-1}+\log |E|+\frac{1}{2} \log (|E|-1)\right)+O\left(p^{3 r}\right) .
\end{aligned}
$$




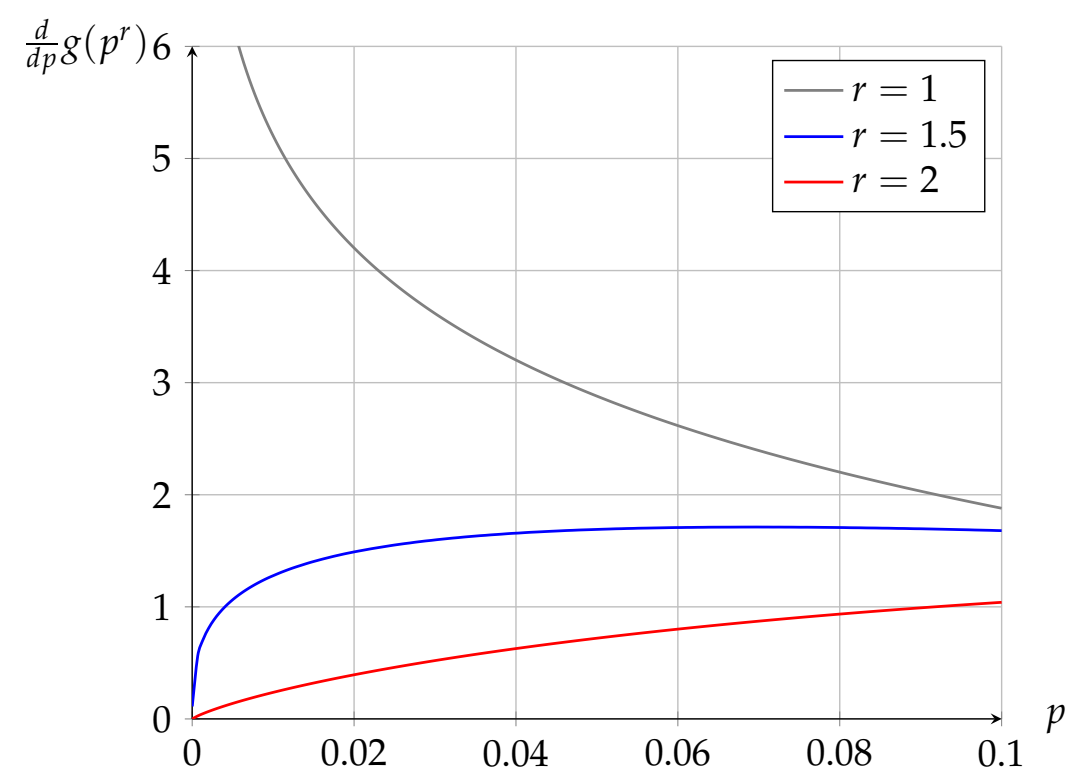

Figure 1: Plot of the derivative of $g\left(p^{r}\right)$ with respect to $p$ for $r \in\{1,1.5,2\}$, where $g(\eta)=-\eta \log \eta$.

For the private capacity, we have the following from Theorem 7:

$$
\left|P(\mathcal{N})-I_{\mathcal{C}}(\mathcal{N})\right| \leq f_{2}(\eta)
$$

where

$$
\begin{aligned}
f_{2}(\eta) & :=\eta \log (|E|-1)+4 \eta \log |E|+2 h\left(\frac{\eta}{2}\right)+4\left(1+\frac{\eta}{2}\right) h\left(\frac{\eta}{2+\eta}\right) \\
& =-3 \eta \log \eta+\left(3+3(\ln 2)^{-1}+\log (|E|-1)+4 \log |E|\right) \eta+O\left(\eta^{2}\right) .
\end{aligned}
$$

In a similar way as above, we can derive the following:

Lemma 10. Let $\mathcal{N}=\mathcal{N}(p)$ be a channel defined in terms of a noise parameter $p \in[0,1]$, and $\operatorname{dg}(\mathcal{N}) \leq$ $c p^{r}$ for some $r>1$ and some constant $c$. Then,

$$
\begin{aligned}
\left|P(\mathcal{N})-I_{c}(\mathcal{N})\right| \leq 3 c r p^{r-1} & (-p \log p) \\
& +c p^{r}\left(-3 \log c+3+3(\ln 2)^{-1}+\log (|E|-1)+4 \log |E|\right)+O\left(p^{2 r}\right) .
\end{aligned}
$$

Our main contribution in this paper is to note that many interesting channels $\mathcal{N}$ satisfying $\| \mathcal{N}-$ id $\|_{\diamond}=O(p)$ have $\operatorname{dg}(\mathcal{N})=O\left(c p^{r}\right)$ for some $r>1$ and a constant $c$, such that Lemma 9 and Lemma 10 apply. In the following subsection, we prove that any channel $\mathcal{N}$ which is $\varepsilon$-close to the identity in the diamond norm has $\operatorname{dg}(\mathcal{N}) \leq 2 \varepsilon^{1.5}$. Furthermore, in Section 4 we improve the exponent to $r=2$ for the Pauli channels.

\subsection{Degrading a low-noise channel with its complementary channel}

Low-noise channels as defined at the beginning of this section are natural examples of "almost" degradable channels, since the identity channel is trivially degradable: its degrading map is given 


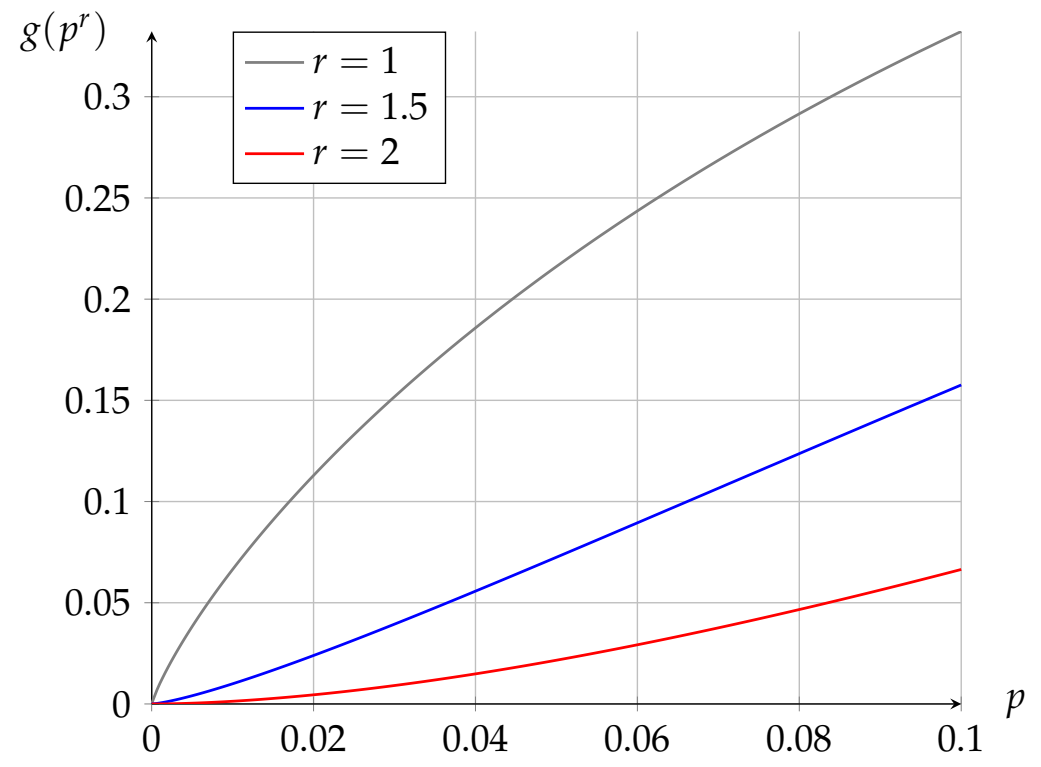

Figure 2: Plot of the function $g\left(p^{r}\right)$ for $r \in\{1,1.5,2\}$, where $g(\eta)=$ $-\eta \log \eta$.

by its complementary channel $\mathrm{id}^{c}(\cdot)=\operatorname{tr}(\cdot)|0\rangle\left\langle\left. 0\right|_{E}\right.$ that outputs a fixed state to the environment. This suggests that the same holds approximately for low-noise channels, i.e., a channel $\mathcal{N}$ that is $\varepsilon$-close to the identity should be approximately degraded by its complementary channel $\mathcal{N}^{c}$. Indeed, one of the main results of this paper, Theorem 11 below, shows that a channel $\mathcal{N}$ with $\| \mathcal{N}-$ id $\|_{\diamond} \leq \varepsilon$ is $2 \varepsilon^{3 / 2}$-approximately degradable with respect to its complementary channel. We prove later (Theorem 15 in Section 4) that the dependence on $\varepsilon$ can be improved to quadratic order for the class of Pauli channels.

Theorem 11. Let $\mathcal{N}$ be a low-noise quantum channel, i.e., $\|\mathcal{N}-\mathrm{id}\|_{\diamond} \leq \varepsilon$ for some $\varepsilon \in[0,2]$. Then $\mathcal{N}$ is $2 \varepsilon^{3 / 2}$-approximately degradable:

$$
\left\|\mathcal{N}^{c}-\mathcal{N}^{c} \circ \mathcal{N}\right\|_{\diamond} \leq 2 \varepsilon^{3 / 2}
$$

Proof. Let $\rho$ be a quantum state achieving the maximum in the definition (1) of the diamond norm of $\mathcal{N}^{c}-\mathcal{N}^{c} \circ \mathcal{N}$, so that

$$
\begin{aligned}
\left\|\mathcal{N}^{c}-\mathcal{N}^{c} \circ \mathcal{N}\right\|_{\diamond} & =\left\|\mathrm{id} \otimes \mathcal{N}^{c}(\rho)-\mathrm{id} \otimes\left(\mathcal{N}^{c} \circ \mathcal{N}\right)(\rho)\right\|_{1} \\
& =\left\|\mathrm{id} \otimes \mathcal{N}^{c}(\delta)\right\|_{1},
\end{aligned}
$$

where $\delta:=\rho-\mathrm{id} \otimes \mathcal{N}(\rho)$. This is a traceless, Hermitian operator that can be expressed as

$$
\delta=\sum_{i} \lambda_{i}\left|\psi_{i}\right\rangle\left\langle\psi_{i}\right|,
$$

where $\sum_{i} \lambda_{i}=0$ since $\delta$ is traceless, and $\sum_{i}\left|\lambda_{i}\right| \leq \varepsilon$ since $\|\mathcal{N}-\mathrm{id}\|_{\diamond} \leq \varepsilon$. Furthermore, we have $\operatorname{tr}_{2} \delta=\operatorname{tr}_{2} \rho-\operatorname{tr}_{2}(\mathrm{id} \otimes \mathcal{N}(\rho))=0$ due to $\mathcal{N}$ being trace-preserving, and hence,

$$
\operatorname{tr}_{2} \delta=\sum_{i} \lambda_{i} \operatorname{tr}_{2}\left|\psi_{i}\right\rangle\left\langle\psi_{i}\right|=0 .
$$

Using the triangle inequality for the trace distance, we bound

$$
\left\|\mathcal{N}^{c}-\mathcal{N}^{c} \circ \mathcal{N}\right\|_{\diamond}=\left\|\operatorname{id} \otimes \mathcal{N}^{c}(\delta)\right\|_{1}
$$




$$
\leq\left\|\mathrm{id} \otimes \mathcal{N}^{c}(\delta)-\mathrm{id} \otimes \mathrm{id}^{c}(\delta)\right\|_{1}+\left\|\mathrm{id} \otimes \mathrm{id}^{c}(\delta)\right\|_{1},
$$

where the complementary channel $\mathrm{id}^{c}$ of the identity map is the completely depolarizing channel

$$
\operatorname{id}^{c}(X)=\operatorname{tr}(X)|0\rangle\langle 0|,
$$

defined in terms of some pure state $|0\rangle$ of the environment of $\mathcal{N}$. For the first term in (13), we have by another application of the triangle inequality that

$$
\begin{aligned}
\left\|\mathrm{id} \otimes \mathcal{N}^{c}(\delta)-\mathrm{id} \otimes \mathrm{id}^{c}(\delta)\right\|_{1} & \leq \sum_{i}\left|\lambda_{i}\right|\left\|\mathrm{id} \otimes \mathcal{N}^{c}\left(\left|\psi_{i}\right\rangle\left\langle\psi_{i}\right|\right)-\mathrm{id} \otimes \operatorname{id}^{c}\left(\left|\psi_{i}\right\rangle\left\langle\psi_{i}\right|\right)\right\|_{1} \\
& \leq 2 \sqrt{\varepsilon} \sum_{i}\left|\lambda_{i}\right| \\
& \leq 2 \varepsilon^{3 / 2}
\end{aligned}
$$

where in the second inequality we used the following bound that holds for all $i$ and follows from Corollary 4:

$$
\left\|\mathrm{id} \otimes \mathcal{N}^{c}\left(\left|\psi_{i}\right\rangle\left\langle\psi_{i}\right|\right)-\mathrm{id} \otimes \mathrm{id}^{c}\left(\left|\psi_{i}\right\rangle\left\langle\psi_{i}\right|\right)\right\|_{1} \leq\left\|\mathcal{N}^{c}-\mathrm{id}^{c}\right\|_{\diamond} \leq 2 \sqrt{\varepsilon} .
$$

For the second term in (13), we have

$$
\mathrm{id} \otimes \mathrm{id}^{c}(\delta)=\sum_{i} \lambda_{i} \operatorname{tr}_{2}\left(\left|\psi_{i}\right\rangle\left\langle\psi_{i}\right|\right) \otimes|0\rangle\langle 0|=0
$$

by (12), and hence, $\left\|\mathrm{id} \otimes \operatorname{id}^{c}(\delta)\right\|_{1}=0$. This concludes the proof.

Combining Theorem 11 with Lemma 9 and 10, we obtain the following main result:

Theorem 12. If $\|\mathcal{N}-\mathrm{id}\|_{\diamond} \leq \varepsilon$ for some $\varepsilon \in[0,2]$, then

$$
\begin{aligned}
& \left|Q(\mathcal{N})-I_{\mathcal{C}}(\mathcal{N})\right| \leq-3 \varepsilon^{1.5} \log \varepsilon+\left((\ln 2)^{-1}+\log |E|+\frac{1}{2} \log (|E|-1)\right) 2 \varepsilon^{1.5}+O\left(\varepsilon^{4.5}\right) \\
& \left|P(\mathcal{N})-I_{\mathcal{c}}(\mathcal{N})\right| \leq-9 \varepsilon^{1.5} \log \varepsilon+\left(3(\ln 2)^{-1}+4 \log |E|+\log (|E|-1)\right) 2 \varepsilon^{1.5}+O\left(\varepsilon^{3}\right) .
\end{aligned}
$$

Recall that $\varepsilon \log \varepsilon$ goes to zero faster than $\varepsilon^{b}$ for any $b<1$. Theorem 12 thus narrows the uncertainty of both capacities to $\varepsilon^{b}$ for $b \approx 1.5$. Furthermore, since the channel $\mathcal{N}$ can already communicate quantum data at the rate $I_{\mathcal{C}}(\mathcal{N})$ using a non-degenerate quantum error correcting code $[17,21,4]$, Theorem 12 shows that degenerate codes only improve quantum communication rates in $O\left(\varepsilon^{b}\right)$ for $b \approx 1.5$. Such a code also transmits private data, and shielding cannot improve the private capacity by the same order.

\section{Generalized Pauli channels}

In this section, we apply our results from Section 3 to the generalized Pauli channels on finite dimension $d$. This class of channels includes the depolarizing channel and the XZ-channel acting on qubits. The quantum and private capacities of these channels have remained unknown for more than 20 years (except for very special extreme values of the noise parameters).

Our main result is that for generalized Pauli channels that are $\varepsilon$-close to the identity channel, the upper bound for the degradability parameter in Theorem 11 can be improved to $O\left(\varepsilon^{2}\right)$. We show 
how a complementary channel with suitably modified noise parameter can be used to achieve such improved approximate degrading.

We start by introducing the generalized Pauli channels on finite dimension $d$. Define the generalized Pauli $X$ and $Z$ operators on $\mathbb{C}^{d}$ :

$$
X|i\rangle=|i+1\rangle, \quad Z|i\rangle=\omega^{i}|i\rangle,
$$

where $\{|i\rangle\}$ is the computational basis, addition is modulo $d$, and $\omega$ is a primitive $d^{\text {th }}$ root of unity. The generalized Pauli basis is given by $G=\left\{X^{k} Z^{l}: 0 \leq k, l \leq d-1\right\}$, and the generalized Pauli channel has the form

$$
\mathcal{N}(\rho)=\sum_{U \in G} p_{U} U \rho U^{+},
$$

where $\left\{p_{U}\right\}_{U \in G}$ is a probability distribution. The above reduces to the Pauli channel in the special case $d=2$,

$$
\mathcal{N}_{\mathbf{p}}(\rho)=p_{0} \rho+p_{1} X \rho X+p_{2} \gamma \rho Y+p_{3} Z \rho Z,
$$

where $X, Y, Z$ are the usual Pauli matrices in $\mathcal{B}\left(\mathbb{C}^{2}\right)$, and $\mathbf{p}=\left(p_{0}, p_{1}, p_{2}, p_{3}\right)$ is a probability distribution.

We first illustrate the main ideas on the simpler depolarizing channel, and then state the general result for the Pauli channel which we prove in Appendix A. Generalization to higher dimensions can be done by expressing the channel input in the basis $G$, and noting that the generalized Pauli channel acts diagonally in this basis. The derivation is straightforward and left as an exercise for the interested readers.

\subsection{Depolarizing channel}

We first consider the qubit depolarizing channel with error $p$ :

$$
\mathcal{D}_{p}(\rho):=(1-p) \rho+\frac{p}{3}(X \rho X+Y \rho Y+Z \rho Z) \text { for } p \in[0,1],
$$

which corresponds to setting $p_{1}=p_{2}=p_{3}=\frac{p}{3}$ in (14). Note that $\|$ id $-\mathcal{D}_{p} \|_{\diamond}=2 p$, which can be seen as follows: The diamond norm distance in (1) is at least $2 p$ by choosing $M$ to be the maximally entangled state, and at most $2 p$ by the feasible solution $Z_{A B}=p \mathcal{J}$ (id) in (2). Theorem 11 then implies that $\mathcal{D}_{p}$ is $2^{2.5} p^{1.5}$-degradable when choosing the complementary channel $\mathcal{D}_{p}^{c}$ of the depolarizing channel with error $p$ as the degrading map. However, solving the SDP (8) numerically shows that $\operatorname{dg}\left(\mathcal{D}_{p}\right) \approx O\left(p^{2}\right)$, which is better than the bound promised by Theorem 11 by half an order.

Here, we derive an analytic proof of the above numerical observation. We will prove that $\operatorname{dg}\left(\mathcal{D}_{p}\right)$ is indeed $O\left(p^{2}\right)$. This is achieved by choosing an approximate degrading map to be the complementary channel $\mathcal{D}_{s}^{c}$, where $s=p+a p^{2}$ for a suitable choice of the parameter $a>0$. To see the intuition, suppose we want $\mathcal{D}_{s}^{c} \circ \mathcal{D}_{p}$ to be close to $\mathcal{D}_{p}^{c}$. Then, choosing $s$ to be slightly larger than $p$ transmits slightly more information to Bob in the output of $\mathcal{D}_{s}^{c}$, which compensates for the slightly worse input to $\mathcal{D}_{s}^{c}$ that is corrupted by $\mathcal{D}_{p}$.

Theorem 13. For $p \approx 0$, we have

$$
\operatorname{dg}\left(\mathcal{D}_{p}\right) \leq \frac{8}{9}(6+\sqrt{2}) p^{2}+O\left(p^{3}\right)
$$


Proof. We first show that, setting $s=p+a p^{2}$, there is a value of $a$ for which $\left\|\mathcal{D}_{s}^{c} \circ \mathcal{D}_{p}-\mathcal{D}_{p}^{c}\right\|_{\diamond} \approx$ $O\left(p^{2}\right)$. Then, for this $s$, we derive two upper bounds to $\left\|\mathcal{D}_{s}^{c} \circ \mathcal{D}_{p}-\mathcal{D}_{p}^{c}\right\|_{\diamond}$ : a rough bound with a simpler derivation showing the idea, and an improved bound with a more complex derivation yielding a better leading constant.

The complementary channel of $\mathcal{D}_{p}$, which we refer to as the epolarizing channel (cf. [13]), can be chosen to be

$$
\mathcal{D}_{p}^{c}(\rho)=\left(\begin{array}{cccc}
(1-p) \operatorname{tr}(\rho) & \sqrt{\frac{p(1-p)}{3}}\langle X, \rho\rangle & \sqrt{\frac{p(1-p)}{3}}\langle Y, \rho\rangle & \sqrt{\frac{p(1-p)}{3}}\langle Z, \rho\rangle \\
\sqrt{\frac{p(1-p)}{3}}\langle X, \rho\rangle & \frac{p}{3} \operatorname{tr}(\rho) & -\frac{i p}{3}\langle Z, \rho\rangle & \frac{i p}{3}\langle Y, \rho\rangle \\
\sqrt{\frac{p(1-p)}{3}}\langle Y, \rho\rangle & \frac{i p}{3}\langle Z, \rho\rangle & \frac{p}{3} \operatorname{tr}(\rho) & -\frac{i p}{3}\langle X, \rho\rangle \\
\sqrt{\frac{p(1-p)}{3}}\langle Z, \rho\rangle & -\frac{i p}{3}\langle Y, \rho\rangle & \frac{i p}{3}\langle X, \rho\rangle & \frac{p}{3} \operatorname{tr}(\rho)
\end{array}\right) .
$$

Using (15) and (16), we further obtain $\mathcal{D}_{s}^{c} \circ \mathcal{D}_{p}(\rho)=$

$$
\left(\begin{array}{cccc}
(1-s) \operatorname{tr}(\rho) & \sqrt{\frac{s(1-s)}{3}}\left(1-\frac{4 p}{3}\right)\langle X, \rho\rangle & \sqrt{\frac{s(1-s)}{3}}\left(1-\frac{4 p}{3}\right)\langle Y, \rho\rangle & \sqrt{\frac{s(1-s)}{3}}\left(1-\frac{4 p}{3}\right)\langle Z, \rho\rangle \\
\sqrt{\frac{s(1-s)}{3}}\left(1-\frac{4 p}{3}\right)\langle X, \rho\rangle & \frac{s}{3} \operatorname{tr}(\rho) & -\frac{i s}{3}\left(1-\frac{4 p}{3}\right)\langle Z, \rho\rangle & \frac{i s}{3}\left(1-\frac{4 p}{3}\right)\langle Y, \rho\rangle \\
\sqrt{\frac{s(1-s)}{3}}\left(1-\frac{4 p}{3}\right)\langle Y, \rho\rangle & \frac{i s}{3}\left(1-\frac{4 p}{3}\right)\langle Z, \rho\rangle & \frac{s}{3} \operatorname{tr}(\rho) & -\frac{i s}{3}\left(1-\frac{4 p}{3}\right)\langle X, \rho\rangle \\
\sqrt{\frac{s(1-s)}{3}}\left(1-\frac{4 p}{3}\right)\langle Z, \rho\rangle & -\frac{i s}{3}\left(1-\frac{4 p}{3}\right)\langle Y, \rho\rangle & \frac{i s}{3}\left(1-\frac{4 p}{3}\right)\langle X, \rho\rangle & \frac{s}{3} \operatorname{tr}(\rho)
\end{array}\right) .
$$

We set $s=p+a p^{2}$ and $\Phi=\mathcal{D}_{p}^{c}-\mathcal{D}_{s}^{c} \circ \mathcal{D}_{p}=\mathcal{D}_{p}^{c}-\mathcal{D}_{p+a p^{2}}^{c} \circ \mathcal{D}_{p}$, which is given by the difference between (16) and (17).

We first show that for some $a,\left\|\mathcal{D}_{s}^{c} \circ \mathcal{D}_{p}-\mathcal{D}_{p}^{c}\right\|_{\diamond} \approx O\left(p^{2}\right)$, and we derive the following upper bound on the degradability parameter $\operatorname{dg}\left(\mathcal{D}_{p}\right)$,

$$
\operatorname{dg}\left(\mathcal{D}_{p}\right) \leq \frac{64}{3} p^{2}+O\left(p^{5 / 2}\right)
$$

To upper bound $\|\Phi\|_{\diamond}$, we apply Lemma 1, which states that

$$
\|\Phi\|_{\diamond} \leq|A||B|^{2}\|\mathcal{J}(\Phi)\|_{\max } \leq 8\|\mathcal{J}(\Phi)\|_{\max }
$$

Hence, we need to evaluate $\|\mathcal{J}(\Phi)\|_{\max }$, where the Choi matrix is given by $\mathcal{J}(\Phi)=\sum_{i, j=0}^{1}|i\rangle\langle j|$ $\otimes \Phi(|i\rangle\langle j|)$. Due to the block structure of the Choi matrix, $\|\mathcal{J}(\Phi)\|_{\max }=\max _{i, j}\|\Phi(|i\rangle\langle j|)\|_{\max }$. To find this maximum, first note that for any $i$ and $j$, the quantities $\operatorname{tr}(|i\rangle\langle j|),|\langle X, \mid i\rangle\langle j \mid\rangle|,|\langle Y, \mid i\rangle\langle j \mid\rangle|$, and $|\langle Z, \mid i\rangle\langle j \mid\rangle|$ can only be 0 or 1 . So, from inspection of the difference between (16) and (17), $\max _{i, j}\|\Phi(|i\rangle\langle j|)\|_{\max }$ is either $s-p=a p^{2}$, or $\frac{1}{3}\left|s-p+\frac{4}{3} p s\right|=\frac{1}{3}\left|a-\frac{4}{3}\right| p^{2}+O\left(p^{3}\right)$, or

$$
c(p):=\sqrt{\frac{p(1-p)}{3}}-\left(1-\frac{4 p}{3}\right) \sqrt{\frac{\left(p+a p^{2}\right)\left(1-p-a p^{2}\right)}{3}} .
$$

Expanding $c(p)$ in a Taylor series around $p=0$ yields

$$
c(p)=\left(\frac{4}{3 \sqrt{3}}-\frac{a}{2 \sqrt{3}}\right) p^{3 / 2}+O\left(p^{5 / 2}\right),
$$


which is $O\left(p^{5 / 2}\right)$ if $a=\frac{8}{3}$. With this choice, $\max _{i, j}\|\Phi(|i\rangle\langle j|)\|_{\max }=a p^{2}=\frac{8}{3} p^{2}$ for sufficiently small $p$. Altogether, $\|\mathcal{J}(\Phi)\|_{\max } \leq \frac{8}{3} p^{2}+O\left(p^{5 / 2}\right)$, and $\|\mathcal{J}(\Phi)\|_{\diamond} \leq \frac{64}{3} p^{2}+O\left(p^{5 / 2}\right)$, which completes the proof of (18).

Finally, to prove the stronger assertion of the theorem,

$$
\operatorname{dg}\left(\mathcal{D}_{p}\right) \leq \frac{8}{9}(6+\sqrt{2}) p^{2}+O\left(p^{3}\right),
$$

we keep the choice $a=\frac{8}{3}$ to enforce that all coefficients of $\Phi=\mathcal{D}_{p}^{c}-\mathcal{D}_{p+a p^{2}}^{c} \circ \mathcal{D}_{p}$ are $O\left(p^{2}\right)$ by Corollary 2. However, we upper bound $\|\Phi\|_{\diamond}$ with a different technique. Since $\Phi$ is a Hermiticitypreserving map, its diamond norm is maximized by a rank-1 state (see for example [33]). Furthermore, since $\mathcal{D}_{p}^{c}$ and $\mathcal{D}_{p+a p^{2}}^{c} \circ \mathcal{D}_{p}$ are jointly covariant under the full unitary group, the diamond norm $\|\Phi\|_{\diamond}$ is maximized by the (normalized) maximally entangled state $\frac{1}{\sqrt{2}}(|00\rangle+|11\rangle)$ [12, Cor. 2.5], and hence,

$$
\|\Phi\|_{\diamond}=\frac{1}{2}\|\mathcal{J}(\Phi)\|_{1}=\frac{1}{2}\left\|\mathcal{J}\left(\mathcal{D}_{p}\right)-\mathcal{J}\left(\mathcal{D}_{p+a p^{2}}^{c} \circ \mathcal{D}_{p}\right)\right\|_{1} .
$$

It follows from (16) and (17) that $\frac{1}{2} \mathcal{J}(\Phi)=\left(\begin{array}{ll}J_{00} & J_{01} \\ J_{10} & J_{11}\end{array}\right)$, where

$$
\begin{aligned}
& J_{00}=\left(\begin{array}{cccc}
\frac{4 p^{2}}{3} & 0 & 0 & \frac{1}{2} c(p) \\
0 & -\frac{4 p^{2}}{9} & -\frac{2}{27} i p^{2}(8 p-3) & 0 \\
0 & \frac{2}{27} i p^{2}(8 p-3) & -\frac{4 p^{2}}{9} & 0 \\
\frac{1}{2} c(p) & 0 & 0 & -\frac{4 p^{2}}{9}
\end{array}\right) \\
& J_{01}=\left(\begin{array}{cccc}
0 & \frac{1}{2} c(p) & \frac{i}{2} c(p) & 0 \\
\frac{1}{2} c(p) & 0 & 0 & -\frac{2}{27} p^{2}(8 p-3) \\
\frac{i}{2} c(p) & 0 & 0 & -\frac{2}{27} i p^{2}(8 p-3) \\
0 & \frac{2}{27} p^{2}(8 p-3) & \frac{2}{27} i p^{2}(8 p-3) & 0
\end{array}\right) \\
& J_{10}=\left(\begin{array}{cccc}
0 & \frac{1}{2} c(p) & -\frac{i}{2} c(p) & 0 \\
\frac{1}{2} c(p) & 0 & 0 & \frac{2}{27} p^{2}(8 p-3) \\
-\frac{i}{2} c(p) & 0 & 0 & -\frac{2}{27} i p^{2}(8 p-3) \\
0 & -\frac{2}{27} p^{2}(8 p-3) & \frac{2}{27} i p^{2}(8 p-3) & 0
\end{array}\right) \\
& J_{11}=\left(\begin{array}{cccc}
\frac{4}{3} p^{2} & 0 & 0 & -\frac{1}{2} c(p) \\
0 & -\frac{4}{9} p^{2} & \frac{2}{27} i p^{2}(8 p-3) & 0 \\
0 & -\frac{2}{27} i p^{2}(8 p-3) & -\frac{4}{9} p^{2} & 0 \\
-\frac{1}{2} c(p) & 0 & 0 & -\frac{4}{9} p^{2}
\end{array}\right),
\end{aligned}
$$

with $c(p)$ as defined in (19). Using the triangle inequality for the trace norm, we get

$$
\frac{1}{2}\|\mathcal{J}(\Phi)\|_{1} \leq\left\|J_{00}\right\|_{1}+\left\|J_{01}\right\|_{1}+\left\|J_{10}\right\|_{1}+\left\|J_{11}\right\|_{1}=: F(p)
$$




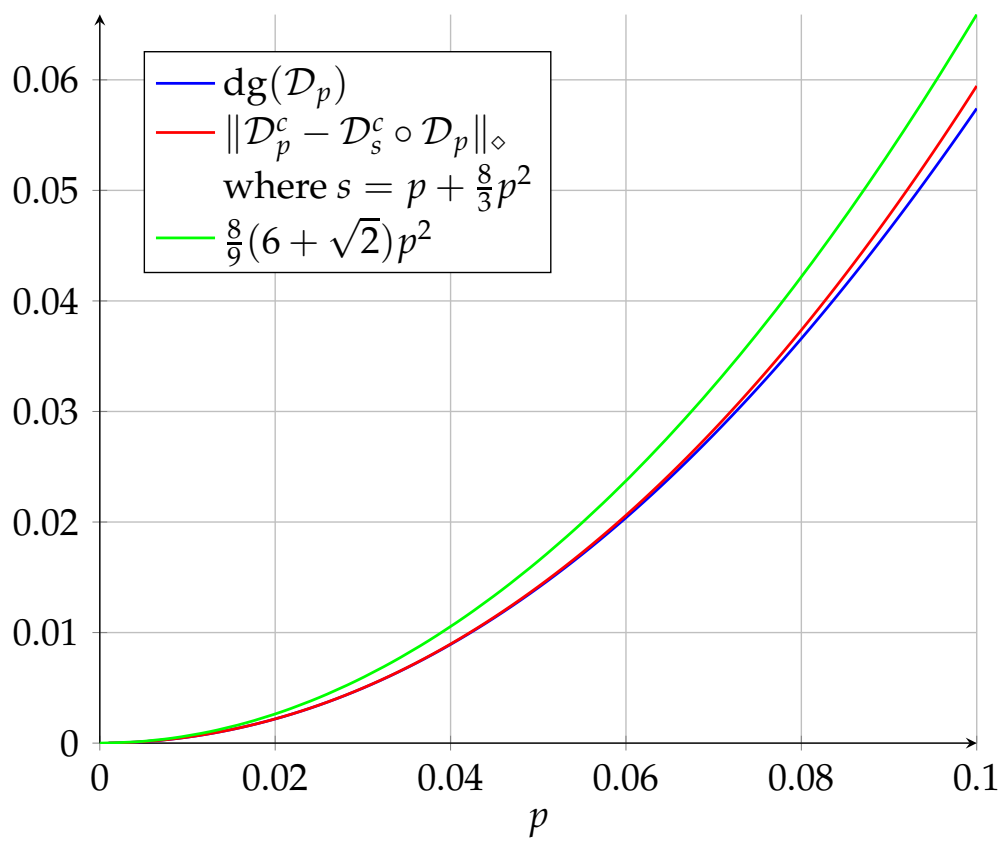

Figure 3: Plot of the optimal degradability parameter $\operatorname{dg}\left(\mathcal{D}_{p}\right)$ (blue) of the qubit depolarizing channel $\mathcal{D}_{p}$ computed using the SDP (8), together with the degradability parameter $\left\|\mathcal{D}_{p}^{c}-\mathcal{D}_{s}^{c} \circ \mathcal{D}_{p}\right\|_{\diamond}($ red) obtained using the degrading map $\mathcal{D}_{s}^{c}$ with $s=p+\frac{8}{3} p^{2}$, and the analytical upper bound $\frac{8}{9}(6+\sqrt{2}) p^{2}$ (green) obtained from Theorem 13.

A Taylor expansion shows that $F(p)=\frac{8}{9}(6+\sqrt{2}) p^{2}+O\left(p^{3}\right)$, from which the bound (20) follows. ${ }^{1}$

In Figure 3 we compare the optimal degradability parameter $\operatorname{dg}\left(\mathcal{D}_{p}\right)$ with the quantity $\| \mathcal{D}_{p}^{c}-$ $\mathcal{D}_{p+a p^{2}}^{c} \circ \mathcal{D}_{p} \|_{\diamond}$ for $a=\frac{8}{3}$, and the analytical upper bound $\frac{8}{9}(6+\sqrt{2}) p^{2}$ obtained from Theorem 13.

Combining Theorem 13 with Lemma 9 and Lemma 10, and using the fact that

$$
I_{\mathcal{C}}\left(\mathcal{D}_{p}\right)=1-h(p)-p \log 3
$$

we obtain the following:

Theorem 14. For small $p$, the quantum and private capacity of the qubit depolarizing channel $\mathcal{D}_{p}$ are given by

$$
\begin{aligned}
& 1-h(p)-p \log 3 \leq Q\left(\mathcal{D}_{p}\right) \leq 1-h(p)-p \log 3-\frac{16}{9}(6+\sqrt{2}) p^{2} \log p+O\left(p^{2}\right) \\
& 1-h(p)-p \log 3 \leq P\left(\mathcal{D}_{p}\right) \leq 1-h(p)-p \log 3-\frac{16}{3}(6+\sqrt{2}) p^{2} \log p+O\left(p^{2}\right) .
\end{aligned}
$$

\footnotetext{
${ }^{1}$ See the Mathematica notebook depol-deg-bound.nb included as an ancillary file on https://arxiv.org/abs/1705.04335.
} 


\subsection{Pauli channels and the XZ-channel}

The above discussion can be extended to all Pauli channels of the form in (14). Note that $\| \mathcal{N}_{\mathbf{p}}-$ id $\|_{\diamond}=2\left(p_{1}+p_{2}+p_{3}\right)$ by an argument similar to the one given for the depolarizing channel. To state our result, we consider a Pauli channel $\mathcal{N}_{\mathbf{p}}$, where the probabilities $p_{i}$ for $i=1,2,3$ are polynomials $p_{i}(p)=c_{i} p+d_{i} p^{2}+\cdots$ in a single parameter $p \in[0,1]$ without constant terms, and $p_{0}=1-p_{1}-p_{2}-p_{3}$. (Note that all Pauli channels can be modeled this way, and the polynomials are not unique.) We now define

$$
\mathbf{s}\left(a_{1}, a_{2}, a_{3}\right):=\left(p_{0}^{\prime}, p_{1}\left(p+a_{1} p^{2}\right), p_{2}\left(p+a_{2} p^{2}\right), p_{3}\left(p+a_{3} p^{2}\right)\right),
$$

where again $p_{0}^{\prime}=1-p_{1}\left(p+a_{1} p^{2}\right)-p_{2}\left(p+a_{2} p^{2}\right)-p_{3}\left(p+a_{3} p^{2}\right){ }^{2}$ We then have the following result, whose proof we give in Appendix A:

Theorem 15. Let $\mathcal{N}_{\mathbf{p}}$ be a generalized Pauli channel with $\mathbf{p}=\left(p_{0}, p_{1}(p), p_{2}(p), p_{3}(p)\right)$, where $p_{0}=$ $1-p_{1}(p)-p_{2}(p)-p_{3}(p)$, and the $p_{i}(p)$ are polynomials in $p$ with $p_{i}(0)=0$ for $i=1,2,3$. Denote by $c_{i}$ the coefficient of $p$ in $p_{i}(p)$ for $i=1,2$, 3. If $c_{i} \neq 0$ then the choices $a_{i}:=4 \sum_{j \neq i} c_{j}$ in (21) ensure that

$$
\left\|\mathcal{N}_{\mathbf{p}}^{c}-\mathcal{N}_{\mathbf{s}\left(a_{1}, a_{2}, a_{3}\right)}^{c} \circ \mathcal{N}_{\mathbf{p}}\right\|_{\diamond}=O\left(p^{2}\right) .
$$

If $c_{i}=0$ for some $i$, then any choice of $a_{i}$ for that $i$ yields (22). Furthermore, we have

$$
\operatorname{dg}\left(\mathcal{N}_{\mathbf{p}}\right) \leq 64\left|c_{1} c_{2}+c_{1} c_{3}+c_{2} c_{3}\right| p^{2}+O\left(p^{3}\right)
$$

Lemma 9 and Lemma 10 now yield the following:

Corollary 16. Let $\mathcal{N}_{\mathbf{p}}$ be a Pauli channel as defined in Theorem 15, and define $C_{\mathbf{p}}:=\left|c_{1} c_{2}+c_{1} c_{3}+c_{2} c_{3}\right|$. The quantum and private capacity of $\mathcal{N}_{\mathbf{p}}$ are given by

$$
\begin{aligned}
& I_{\mathcal{C}}\left(\mathcal{N}_{\mathbf{p}}\right) \leq Q(\mathcal{N}) \leq I_{\mathcal{C}}\left(\mathcal{N}_{\mathbf{p}}\right)-128 C_{\mathbf{p}} p^{2} \log p+O\left(p^{2}\right) \\
& I_{\mathcal{C}}\left(\mathcal{N}_{\mathbf{p}}\right) \leq P(\mathcal{N}) \leq I_{\mathcal{C}}\left(\mathcal{N}_{\mathbf{p}}\right)-384 C_{\mathbf{p}} p^{2} \log p+O\left(p^{2}\right)
\end{aligned}
$$

Theorem 15 and Corollary 16 include the (weaker) result from Section 4.1 about the depolarizing channel, for which we have $c_{i}=\frac{1}{3}$ for $i=1,2,3$, and hence $a_{i}=\frac{8}{3}$ and $C_{\mathbf{p}}=\frac{1}{3}$.

Another interesting example in the class of generalized Pauli channels is the XZ-channel

$$
\mathcal{N}_{p, q}^{X Z}(\rho):=(1-p)(1-q) \rho+p(1-q) X \rho X+p q Y \rho Y+(1-p) q Z \rho Z
$$

that implements independent $X$ and $Z$ errors by applying an $X$-dephasing with probability $p$, and a Z-dephasing with probability $q$. For our discussion, we set $p=q$ and denote the resulting $X Z$-channel by $\mathcal{C}_{p}$,

$$
\mathcal{C}_{p}(\rho)=(1-p)^{2} \rho+\left(p-p^{2}\right) X \rho X+p^{2} Y \rho Y+\left(p-p^{2}\right) Z \rho Z
$$

Thus, we have $c_{1}=1, c_{2}=0, c_{3}=1, d_{1}=-1, d_{2}=1$, and $d_{3}=-1$. Hence, the choices $a_{1}=a_{2}=a_{3}=4$ ensure (22) by Theorem 15, and from (23) we obtain the analytic bound $\operatorname{dg}\left(\mathcal{C}_{p}\right) \leq$ $64 p^{2}+O\left(p^{3}\right)$. However, similar to Theorem 13, we can further improve the bound on $\operatorname{dg}\left(\mathcal{C}_{p}\right)$ :

\footnotetext{
${ }^{2}$ Note that $p_{i}\left(p+a_{i} p^{2}\right)$ denote the polynomial $p_{i}$ with argument $p+a_{i} p^{2}$, not the product of $p_{i}$ and $p+a_{i} p^{2}$.
} 
Theorem 17. For $p \approx 0$, we have

$$
\operatorname{dg}\left(\mathcal{C}_{p}\right) \leq 16 p^{2}+32 p^{5 / 2}+O\left(p^{3}\right)
$$

Proof. For the XZ-channel $\mathcal{C}_{p}$, we have $p_{0}=(1-p)^{2}, p_{1}=p_{3}=p-p^{2}$, and $p_{2}=p^{2}$ by (24). Furthermore, as in the discussion above we choose $s=p+4 p^{2}$, and set $q_{0}=(1-s)^{2}, q_{1}=q_{3}=$ $s-s^{2}$, and $q_{2}=s^{2}$, such that the map $\Phi=\mathcal{C}_{p}^{c}-\mathcal{C}_{s}^{c} \circ \mathcal{C}_{p}$ as given in (27) has coefficients that are $O\left(p^{2}\right)$ by Corollary 2. Since $\Phi$ is Hermiticity-preserving, its diamond norm is maximized by a pure state [33], and since both $\mathcal{C}_{p}^{c}$ and $\mathcal{C}_{s}^{c} \circ \mathcal{C}_{p}$ are covariant with respect to the Pauli group, a 1-design, this pure state can be chosen to be the maximally entangled state $\frac{1}{2}(|00\rangle+|11\rangle)$ [12, Cor. 2.5]. Hence,

$$
\left\|\mathcal{C}_{p}^{c}-\mathcal{C}_{s}^{c} \circ \mathcal{C}_{p}\right\|_{\diamond}=\|\Phi\|_{\diamond}=\frac{1}{2}\|\mathcal{J}(\Phi)\|_{1}
$$

Exploiting the block structure $\frac{1}{2} \mathcal{J}(\Phi)=\left(\begin{array}{ll}G_{00} & G_{01} \\ G_{10} & G_{11}\end{array}\right)$ that follows from (27) together with the triangle inequality for the trace norm, we obtain the upper bound

$$
\operatorname{dg}\left(\mathcal{C}_{p}\right) \leq\left\|\mathcal{C}_{p}^{c}-\mathcal{C}_{s}^{c} \circ \mathcal{C}_{p}\right\|_{\diamond}=\frac{1}{2}\|\mathcal{J}(\Phi)\|_{1} \leq\left\|G_{00}\right\|_{1}+\left\|G_{11}\right\|_{1}+\left\|G_{01}\right\|_{1}+\left\|G_{10}\right\|_{1}
$$

and a Taylor expansion of the right-hand side of (25) shows that $\operatorname{dg}\left(\mathcal{C}_{p}\right) \leq 16 p^{2}+32 p^{5 / 2}+O\left(p^{3}\right)$, which proves the claim. ${ }^{3}$

In Figure 4 we compare the optimal degradability parameter $\operatorname{dg}\left(\mathcal{C}_{p}\right)$ with the quantity $\| \mathcal{C}_{p}^{c}-\mathcal{C}_{s}^{c} \circ$ $\mathcal{C}_{p} \|_{\diamond}$ and the analytical upper bound $16 p^{2}+32 p^{5 / 2}$ obtained from Theorem 17.

Numerics suggest that the coherent information $I_{\mathcal{c}}\left(\mathcal{C}_{p}\right)$ is achieved by a completely mixed state $\pi=I / 2$, giving

$$
I_{c}\left(\mathcal{C}_{p}\right)=I_{c}\left(\pi ; \mathcal{C}_{p}\right)=1-2 h(p) .
$$

Putting Corollary 16 and Theorem 17 together, and using the above coherent information expression, we obtain

Corollary 18. For small $p$, the quantum and private capacity of the XZ-channel $\mathcal{C}_{p}=\mathcal{N}_{p, p}^{X Z}$ with equal $X$-and Z-dephasing probability are given by

$$
\begin{aligned}
& 1-2 h(p) \leq Q\left(\mathcal{C}_{p}\right) \leq 1-2 h(p)-32 p^{2} \log p+O\left(p^{2}\right) \\
& 1-2 h(p) \leq P\left(\mathcal{C}_{p}\right) \leq 1-2 h(p)-96 p^{2} \log p+O\left(p^{2}\right) .
\end{aligned}
$$

\section{Conclusion}

Our results can be extended to cover generalized low-noise channels $\mathcal{N}$, for which there exists another channel $\mathcal{M}$ such that $\|\mathcal{M} \circ \mathcal{N}-I\|_{\diamond} \leq \epsilon$. For example, this includes all channels that are close to isometric channels. For a generalized low-noise channel, we have by Theorem 11 that

$$
\left\|(\mathcal{M} \circ \mathcal{N})^{c}-(\mathcal{M} \circ \mathcal{N})^{c} \circ(\mathcal{M} \circ \mathcal{N})\right\|_{\diamond} \leq 2 \epsilon^{3 / 2} .
$$

\footnotetext{
${ }^{3}$ We refer to the Mathematica notebook $\mathrm{xz}$-deg-bound.nb included in the ancillary files of https://arxiv.org/abs/1705.04335 for details.
} 


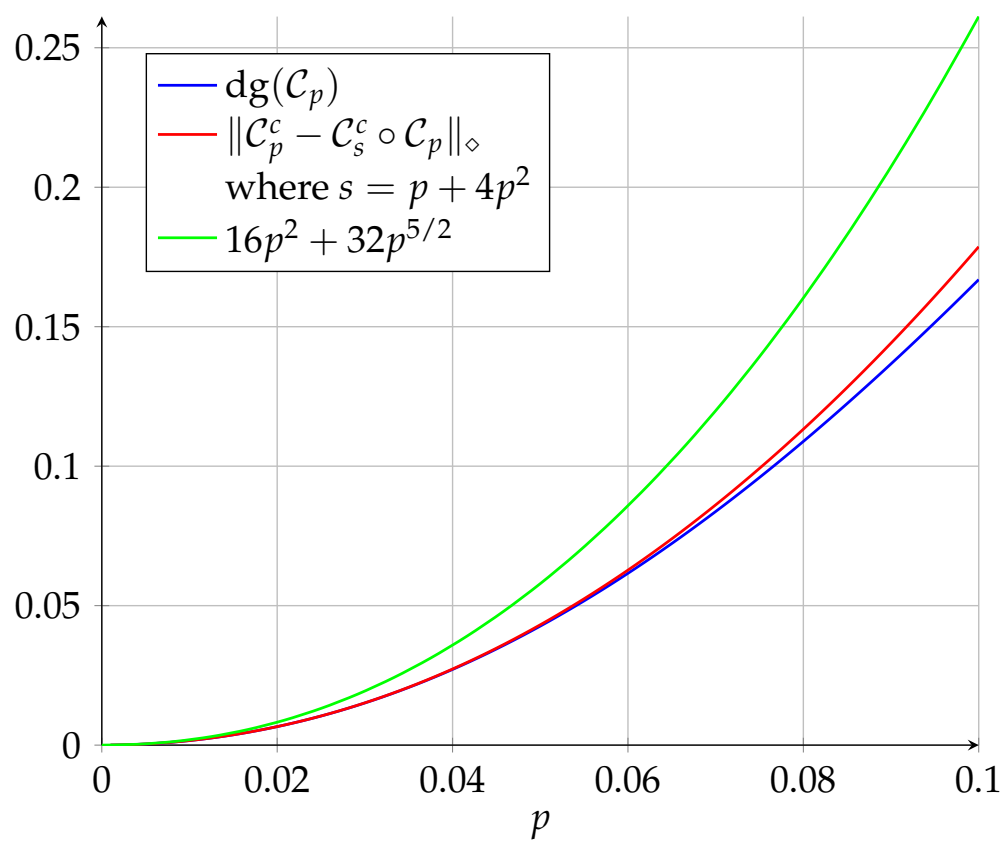

Figure 4: Plot of the optimal degradability parameter $\operatorname{dg}\left(\mathcal{C}_{p}\right)$ (blue) of the $X Z$-channel $\mathcal{C}_{p}$ computed using the SDP (8), together with the degradability parameter $\left\|\mathcal{C}_{p}^{c}-\mathcal{C}_{s}^{c} \circ \mathcal{C}_{p}\right\|_{\diamond}$ (red) obtained using the degrading map $\mathcal{C}_{s}^{c}$ with $s=p+4 p^{2}$, and the analytical upper bound $16 p^{2}+32 p^{5 / 2}$ (green) obtained from Theorem 17.

Furthermore, up to an isometry,

$$
(\mathcal{M} \circ \mathcal{N})^{c}(\rho)=\left(\mathcal{M}^{c} \otimes I_{E_{1}}\right)\left(U_{\mathcal{N}} \rho U_{\mathcal{N}}^{\dagger}\right),
$$

where $U_{\mathcal{N}}: A \rightarrow B E_{1}$ is an isometric extension of $\mathcal{N}$ and $\mathcal{M}^{c}: B \rightarrow E_{2}$, so that $\operatorname{tr}_{E_{2}}(\mathcal{M} \circ \mathcal{N})^{c}(\rho)=$ $\mathcal{N}^{c}(\rho)$. Equation 26 therefore implies

$$
\left\|\mathcal{N}^{c}-\operatorname{tr}_{E_{2}}(\mathcal{M} \circ \mathcal{N})^{c} \circ(\mathcal{M} \circ \mathcal{N})\right\|_{\diamond} \leq 2 \epsilon^{3 / 2},
$$

so that letting $\mathcal{D}=\operatorname{tr}_{E_{2}}(\mathcal{M} \circ \mathcal{N})^{c} \circ \mathcal{M}$ we have $\left\|\mathcal{N}^{c}-\mathcal{D} \circ \mathcal{N}\right\|_{\diamond} \leq 2 \epsilon^{3 / 2}$ and $\mathcal{N}$ has degradability parameter no bigger than $2 \epsilon^{3 / 2}$. We thus find that the same bounds as in Theorem 12 apply in the case of a generalized low-noise channel $\mathcal{N}$.

We conclude with some implications of our results. The quantum and private capacities of a quantum channel are intractable to calculate in general. This is because nonadditivity effects require us to regularize the coherent information and private information to obtain the quantum and private capacity, respectively. We have shown that for low-noise channels, for which $\|\mathcal{N}-\mathrm{id}\|_{\diamond} \leq \varepsilon$, such nonadditivity effects are negligible. In particular, we find that both the private and quantum capacities of these channels are given by the one-shot coherent information $I_{\mathcal{C}}(\mathcal{N})$, up to corrections of order $\varepsilon^{1.5} \log \varepsilon$. Furthermore, for the qubit depolarizing channel $\mathcal{D}_{p}$ we obtain even tighter bounds for both the quantum and private capacities:

$$
\begin{aligned}
& 1-h(p)-p \log 3 \leq Q\left(\mathcal{D}_{p}\right) \leq 1-h(p)-p \log 3-\frac{16}{9}(6+\sqrt{2}) p^{2} \log p+O\left(p^{2}\right) \\
& 1-h(p)-p \log 3 \leq P\left(\mathcal{D}_{p}\right) \leq 1-h(p)-p \log 3-\frac{16}{3}(6+\sqrt{2}) p^{2} \log p+O\left(p^{2}\right),
\end{aligned}
$$


and similar results hold for all generalized Pauli channels in dimension $d$. Our key new finding is that channels within $\varepsilon$ of perfect are also exceptionally close to degradable, with degradability parameter of $O\left(\varepsilon^{1.5}\right)$ in general and $O\left(p^{2}\right)$ for generalized Pauli channels.

The nonadditivty of coherent information for a general channel implies that degenerate codes are sometimes needed to achieve the quantum capacity [6, 24, 8, 26, 25, 3], but little is known about these codes despite 20 years of research. Having shown that the coherent information is essentially the quantum capacity for low-noise channels, we have also arrived at a refreshing result that using random block codes on the typical subspace of the optimal input (for the 1-shot coherent information) essentially achieves the capacity.

Likewise, our findings have implications in quantum cryptography. In quantum key distribution, quantum states are transmitted through well-characterized noisy quantum channels that are subject to further adversarial attacks. Parameter estimation is used to determine the effective channel (see for example [19]) and the optimal key rate of one-way quantum key distribution is the private capacity of the effective channel. These effective channels typically have low noise (e.g., $1-2 \%$ in [28]), and our results imply near-optimality of the simple classical error correction and privacy amplification procedures that approach the one-shot coherent information of the effective channel. In particular, for the XZ-channel with bit-flip probability $p$, the optimal key rate is $1-2 h(p)+O\left(p^{2} \log p\right)$.

\section{Acknowledgements}

We thank Charles Bennett, Ke Li, John Smolin, and John Watrous for inspiring discussions, and Mark M. Wilde for helpful feedback. DL is further supported by NSERC and CIFAR, and FL and GS by the National Science Foundation under Grant Number 1125844.

\section{A Proof of Theorem 15}

In this appendix we prove Theorem 15, which states that for any generalized Pauli channel

$$
\mathcal{N}_{\mathbf{p}}(\rho)=p_{0} \rho+p_{1} X \rho X+p_{2} \gamma \rho Y+p_{3} Z \rho Z,
$$

for which the coefficients $p_{i}(p)=c_{i} p+d_{i} p^{2}+\cdots$ are polynomials with zero constant term in a parameter $p$, there is a choice of $a_{1}, a_{2}, a_{3}$ such that

$$
\left\|\mathcal{N}_{\mathbf{p}}^{c}-\mathcal{N}_{\mathbf{s}\left(a_{1}, a_{2}, a_{3}\right)}^{c} \circ \mathcal{N}_{\mathbf{p}}\right\|_{\diamond}=O\left(p^{2}\right) .
$$

Here, we define $\mathbf{s}\left(a_{1}, a_{2}, a_{3}\right):=\left(p_{0}^{\prime}, p_{1}\left(p+a_{1} p^{2}\right), p_{2}\left(p+a_{2} p^{2}\right), p_{3}\left(p+a_{3} p^{2}\right)\right)$, with $p_{0}^{\prime}$ chosen such that $\mathbf{s}\left(a_{1}, a_{2}, a_{3}\right)$ is a probability distribution.

An isometric extension of the Pauli channel is given by

$$
V_{\mathbf{p}}:=\sqrt{p_{0}} \mathbb{1} \otimes|0\rangle_{E}+\sqrt{p_{1}} X \otimes|1\rangle_{E}+\sqrt{p_{2}} Y \otimes|2\rangle_{E}+\sqrt{p_{3}} Z \otimes|3\rangle_{E},
$$

where $\left\{|0\rangle_{E},|1\rangle_{E},|2\rangle_{E},|3\rangle_{E}\right\}$ is an orthonormal basis for the environment $E$. For an arbitrary linear 
operator $\rho$, it is straightforward to find that (see e.g. [13])

$$
\mathcal{N}_{\mathbf{p}}^{c}(\rho)=\operatorname{tr}_{B}\left(V_{\mathbf{p}} \rho V_{\mathbf{p}}^{\dagger}\right)=\left(\begin{array}{cccc}
p_{0} \operatorname{tr}(\rho) & \sqrt{p_{0} p_{1}}\langle X, \rho\rangle & \sqrt{p_{0} p_{2}}\langle Y, \rho\rangle & \sqrt{p_{0} p_{3}}\langle Z, \rho\rangle \\
\sqrt{p_{0} p_{1}}\langle X, \rho\rangle & p_{1} \operatorname{tr}(\rho) & -i \sqrt{p_{1} p_{2}}\langle Z, \rho\rangle & i \sqrt{p_{1} p_{3}}\langle Y, \rho\rangle \\
\sqrt{p_{0} p_{2}}\langle Y, \rho\rangle & i \sqrt{p_{1} p_{2}}\langle Z, \rho\rangle & p_{2} \operatorname{tr}(\rho) & -i \sqrt{p_{2} p_{3}}\langle X, \rho\rangle \\
\sqrt{p_{0} p_{3}}\langle Z, \rho\rangle & -i \sqrt{p_{1} p_{3}}\langle Y, \rho\rangle & i \sqrt{p_{2} p_{3}}\langle X, \rho\rangle & p_{3} \operatorname{tr}(\rho)
\end{array}\right) .
$$

Writing $\mathbf{q}=\left(q_{0}, q_{1}, q_{2}, q_{3}\right)$, the action of the map $\Phi:=\mathcal{N}_{\mathbf{p}}^{c}-\mathcal{N}_{\mathbf{q}}^{c} \circ \mathcal{N}_{\mathbf{p}}$ on a linear operator $\rho$ is

$$
\Phi(\rho)=\left(\begin{array}{cccc}
\left(p_{0}-q_{0}\right) \operatorname{tr}(\rho) & t_{1}\langle X, \rho\rangle & t_{2}\langle Y, \rho\rangle & t_{3}\langle Z, \rho\rangle \\
t_{1}\langle X, \rho\rangle & \left(p_{1}-q_{1}\right) \operatorname{tr}(\rho) & -i u_{3}\langle Z, \rho\rangle & i u_{2}\langle Y, \rho\rangle \\
t_{2}\langle Y, \rho\rangle & i u_{3}\langle Z, \rho\rangle & \left(p_{2}-q_{2}\right) \operatorname{tr}(\rho) & -i u_{1}\langle X, \rho\rangle \\
t_{3}\langle Z, \rho\rangle & -i u_{2}\langle Y, \rho\rangle & i u_{1}\langle X, \rho\rangle & \left(p_{3}-q_{3}\right) \operatorname{tr}(\rho)
\end{array}\right)
$$

with the following coefficients:

$$
\begin{aligned}
& t_{1}=\sqrt{p_{0} p_{1}}-\sqrt{q_{0} q_{1}}\left(p_{0}+p_{1}-p_{2}-p_{3}\right) \\
& t_{2}=\sqrt{p_{0} p_{2}}-\sqrt{q_{0} q_{2}}\left(p_{0}-p_{1}+p_{2}-p_{3}\right) \\
& t_{3}=\sqrt{p_{0} p_{3}}-\sqrt{q_{0} q_{3}}\left(p_{0}-p_{1}-p_{2}+p_{3}\right) \\
& u_{1}=\sqrt{p_{2} p_{3}}-\sqrt{q_{2} q_{3}}\left(p_{0}+p_{1}-p_{2}-p_{3}\right) \\
& u_{2}=\sqrt{p_{1} p_{3}}-\sqrt{q_{1} q_{3}}\left(p_{0}-p_{1}+p_{2}-p_{3}\right) \\
& u_{3}=\sqrt{p_{1} p_{2}}-\sqrt{q_{1} q_{2}}\left(p_{0}-p_{1}-p_{2}+p_{3}\right) .
\end{aligned}
$$

Similar to the case for the depolarizing channel, $\|\mathcal{J}(\Phi)\|_{\max }$ is the maximum among $\left|p_{i}-q_{i}\right|$ for $i=0,1,2,3$ and $t_{i}, u_{i}$ for $i=1,2,3$.

We choose $q_{i}(p)=p_{i}\left(p+a_{i} p^{2}\right)$ for $i=1,2,3$, and $q_{0}=1-q_{1}-q_{2}-q_{3}$. It follows that $p_{i}-q_{i}=$ $O\left(p^{2}\right)$ for $i=0,1,2,3$. Similarly, expanding the $u_{i}$ in a Taylor series around $p=0$ shows that $u_{i}=O\left(p^{2}\right)$ for any choices of $a_{i}$. Hence, it remains to check the coefficients $t_{i}$, which we again expand in a Taylor series around $p=0$ :

$$
\begin{aligned}
& t_{1}=\frac{\sqrt{c_{1}}}{2}\left(a_{1}-4\left(c_{2}+c_{3}\right)\right) p^{3 / 2}+O\left(p^{5 / 2}\right) \\
& t_{2}=\frac{\sqrt{c_{2}}}{2}\left(a_{2}-4\left(c_{1}+c_{3}\right)\right) p^{3 / 2}+O\left(p^{5 / 2}\right) \\
& t_{3}=\frac{\sqrt{c_{3}}}{2}\left(a_{3}-4\left(c_{1}+c_{2}\right)\right) p^{3 / 2}+O\left(p^{5 / 2}\right) .
\end{aligned}
$$

We see that if $c_{i}=0$, then the coefficient of $p^{3 / 2}$ in $t_{i}$ vanishes for any choice of $a_{i}$. If on the other hand $c_{i} \neq 0$, then the coefficient of $p^{3 / 2}$ in $t_{i}$ vanishes upon choosing $a_{i}=4 \sum_{j \neq i} c_{j}$.

In summary, for the above choices of $a_{1}, a_{2}, a_{3}$, the max norm $\|\mathcal{J}(\Phi)\|_{\max }$ is the maximum of $\mid p_{i}-$ $q_{i} \mid$ for $i=0,1,2,3$ and $u_{i}$ for $i=1,2,3$. Recall that

$$
\begin{aligned}
& p_{i}(p)=c_{i} p+d_{i} p^{2}+O\left(p^{3}\right) \\
& q_{i}(p)=p_{i}\left(p+a_{i} p^{2}\right)=c_{i} p+\left(a_{i} c_{i}+d_{i}\right) p^{2}+O\left(p^{3}\right) .
\end{aligned}
$$


Hence, since $p \approx 0$,

$$
\begin{aligned}
\max _{i}\left|p_{i}-q_{i}\right| & =\left|p_{0}-q_{0}\right| \\
& =\left|\sum\left(p_{i}-q_{i}\right)\right| \\
& =\left|\sum a_{i} c_{i}\right| p^{2}+O\left(p^{3}\right) \\
& =8\left|c_{1} c_{2}+c_{1} c_{3}+c_{2} c_{3}\right| p^{2}+O\left(p^{3}\right),
\end{aligned}
$$

where we substituted $a_{i}=4 \sum_{j \neq i} c_{j}$ in the last line. Expanding $u_{i}$ for $p \approx 0$ gives

$$
\begin{aligned}
& u_{1}=-4 c_{1} \sqrt{c_{2} c_{3}} p^{2}+O\left(p^{3}\right) \\
& u_{2}=-4 c_{2} \sqrt{c_{1} c_{3}} p^{2}+O\left(p^{3}\right) \\
& u_{3}=-4 c_{3} \sqrt{c_{1} c_{2}} p^{2}+O\left(p^{3}\right) .
\end{aligned}
$$

From (28) and (29), we infer

$$
\|\mathcal{J}(\Phi)\|_{\max }=\left|p_{0}-q_{0}\right|=8\left|c_{1} c_{2}+c_{1} c_{3}+c_{2} c_{3}\right| p^{2}+O\left(p^{3}\right),
$$

so that by Lemma 1 we have $\|\Phi\|_{\diamond} \leq 64\left|c_{1} c_{2}+c_{1} c_{3}+c_{2} c_{3}\right| p^{2}+O\left(p^{3}\right)$, which concludes the proof of Theorem 15.

\section{References}

[1] D. Bruß et al. "Optimal universal and state-dependent quantum cloning". Physical Review A 57 (4 1998), pp. 2368-2378. arXiv: quant-ph / 9705038.

[2] A. Cross, K. Li, and G. Smith. "Uniform Additivity in Classical and Quantum Information". Physical Review Letters 118.4 (2017), p. 040501. arXiv: 1601.05434 [quant-ph].

[3] T. Cubitt et al. "Unbounded number of channel uses may be required to detect quantum capacity". Nature Communications 6 (2015). arXiv: 1408.5115 [quant-ph] .

[4] I. Devetak. "The private classical capacity and quantum capacity of a quantum channel". IEEE Transactions on Information Theory 51.1 (2005), pp. 44-55. arXiv: quant-ph/ 0304127.

[5] I. Devetak and P. W. Shor. "The capacity of a quantum channel for simultaneous transmission of classical and quantum information". Communications in Mathematical Physics 256.2 (2005), pp. 287-303. arXiv: quant-ph/0311131 [quant-ph].

[6] D. DiVincenzo, P. Shor, and J. Smolin. "Quantum-channel capacity of very noisy channels". Physical Review A 57.2 (1998), pp. 830-839. arXiv: quant-ph/ 9706061.

[7] M. Fekete. "Über die Verteilung der Wurzeln bei gewissen algebraischen Gleichungen mit ganzzahligen Koeffizienten". Mathematische Zeitschrift 17.1 (1923), pp. 228-249.

[8] J. Fern and K. B. Whaley. "Lower bounds on the nonzero capacity of Pauli channels". Physical Review A 78.6 (2008), p. 062335. arXiv: 0708.1597 [quant-ph] .

[9] C. W. Helstrom. "Quantum detection and estimation theory". Journal of Statistical Physics 1.2 (1969), pp. 231-252.

[10] K. Horodecki, M. Horodecki, P. Horodecki, and J. Oppenheim. "Secure key from bound entanglement". Physical Review Letters 94.16 (2005), p. 160502. arXiv: quant-ph/ 0309110. 
[11] D. Kretschmann, D. Schlingemann, and R. F. Werner. "The information-disturbance tradeoff and the continuity of Stinespring's representation". IEEE Transactions on Information Theory 54.4 (2008), pp. 1708-1717. arXiv: quant-ph / 0605009.

[12] F. Leditzky, E. Kaur, N. Datta, and M. M. Wilde. "Approaches for approximate additivity of the Holevo information of quantum channels". arXiv preprint (2017). arXiv: 1709.01111 [quant-ph] .

[13] D. W. Leung and J. Watrous. "On the complementary quantum capacity of the depolarizing channel". arXiv preprint (2015). arXiv: 1510.01366 [quant-ph] .

[14] D. Leung and G. Smith. "Continuity of Quantum Channel Capacities". Communications in Mathematical Physics 292.1 (2009), pp. 201-215. arXiv: 0810.4931 [quant-ph].

[15] D. Leung, K. Li, G. Smith, and J. A. Smolin. "Maximal privacy without coherence". Physical Review Letters 113.3 (2014), p. 030502. arXiv: 1312 . 4989 [quant-ph] .

[16] K. Li, A. Winter, X. Zou, and G. Guo. "Private capacity of quantum channels is not additive". Physical Review Letters 103.12 (2009), p. 120501. arXiv: 0903.4308 [quant-ph].

[17] S. Lloyd. "Capacity of the noisy quantum channel". Physical Review A 55 (1997), pp. 16131622. arXiv: quant-ph/9604015.

[18] M. A. Nielsen and I. L. Chuang. Quantum computation and quantum information. Cambridge: Cambridge University Press, 2000.

[19] R. Renner, N. Gisin, and B. Kraus. "Information-theoretic security proof for quantum-keydistribution protocols". Physical Review A 72.1 (2005), p. 012332. arXiv: quant-ph / 0502064.

[20] C. Shannon. "A mathematical theory of communication". The Bell System Technical Journal 27 (1948), pp. 379-423.

[21] P. Shor. The quantum channel capacity and coherent information. Lecture notes, MSRI Workshop on Quantum Computation. 2002.

[22] G. Smith. "Private classical capacity with a symmetric side channel and its application to quantum cryptography". Physical Review A 78.2 (2008), p. 022306. arXiv: 0705.3838 [ quant-ph].

[23] G. Smith, J. M. Renes, and J. A. Smolin. "Structured codes improve the Bennett-Brassard-84 quantum key rate". Physical Review Letters 100.17 (2008), p. 170502. arXiv: quant-ph / 0607018.

[24] G. Smith and J. A. Smolin. "Degenerate quantum codes for Pauli channels". Physical Review Letters 98.3 (2007), p. 030501. arXiv: quant-ph / 0604107.

[25] G. Smith and J. A. Smolin. "Extensive nonadditivity of privacy". Physical Review Letters 103.12 (2009), p. 120503. arXiv: 0904.4050 [quant-ph].

[26] G. Smith and J. Yard. “Quantum communication with zero-capacity channels". Science 321.5897 (2008), pp. 1812-1815. arXiv: 0807.4935 [quant-ph].

[27] W. F. Stinespring. "Positive functions on $C^{*}$-algebras". Proceedings of the American Mathematical Society 6.2 (1955), pp. 211-216.

[28] D. Stucki et al. "High rate, long-distance quantum key distribution over $250 \mathrm{~km}$ of ultra low loss fibres". New Journal of Physics 11.7 (2009), p. 075003. arXiv: 0903.3907 [ quant-ph ] .

[29] D. Sutter, V. B. Scholz, A. Winter, and R. Renner. "Approximate degradable quantum channels". arXiv preprint (2015). arXiv: 1412.0980 [quant-ph].

[30] S. Watanabe. "Private and Quantum Capacities of More Capable and Less Noisy Quantum Channels". Physical Review A 85 (2012), p. 012326. arXiv: 1110.5746 [quant-ph ]. 
[31] J. Watrous. "Semidefinite programs for completely bounded norms". arXiv preprint (2009). arXiv: 0901.4709 [quant-ph].

[32] J. Watrous. "Simpler semidefinite programs for completely bounded norms". arXiv preprint (2012). arXiv: 1207.5726 [quant-ph].

[33] J. Watrous. Theory of Quantum Information. Cambridge University Press (to be published), 2017. Available online at: https://cs . uwaterloo.ca/ watrous/TQI/.

[34] J. Yard, P. Hayden, and I. Devetak. "Capacity theorems for quantum multiple-access channels: Classical-quantum and quantum-quantum capacity regions". IEEE Transactions on Information Theory 54.7 (2008), pp. 3091-3113. arXiv: quant-ph / 0501045. 